QUARTERLY OF APPLIED MATHEMATICS

VOLUME LXX, NUMBER 1

MARCH 2012, PAGES 133-157

S 0033-569X(2011)01246-9

Article electronically published on September 9, 2011

\title{
QUALITATIVE PROPERTIES OF SOLUTIONS TO A TIME-SPACE FRACTIONAL EVOLUTION EQUATION
}

\author{
BY
}

AHMAD Z. FINO (LaMA-Liban, Lebanese University, P.O. Box 37 Tripoli, Lebanon)

AND

MOKHTAR KIRANE (Département de Mathématiques, Pôle Sciences et Technologies, Université de la Rochelle, Avenue M. Crépeau, La Rochelle 17042, France)

Abstract. In this article, we analyze a spatio-temporally nonlocal nonlinear parabolic equation. First, we validate the equation by an existence-uniqueness result. Then, we show that blowing-up solutions exist and study their time blow-up profile. Also, a result on the existence of global solutions is presented. Furthermore, we establish necessary conditions for local or global existence.

1. Introduction. In this paper, we investigate the spatio-temporally nonlinear parabolic equation

$$
\begin{cases}u_{t}+(-\Delta)^{\beta / 2} u=\frac{1}{\Gamma(1-\gamma)} \int_{0}^{t}(t-s)^{-\gamma}|u|^{p-1} u(s) d s & x \in \mathbb{R}^{N}, t>0 \\ u(x, 0)=u_{0}(x) & x \in \mathbb{R}^{N},\end{cases}
$$

where $u_{0} \in C_{0}\left(\mathbb{R}^{N}\right), N \geq 1,0<\beta \leq 2,0<\gamma<1, p>1$ and the nonlocal operator $(-\Delta)^{\beta / 2}$ is defined by

$$
(-\Delta)^{\beta / 2} v(x):=\mathcal{F}^{-1}\left(|\xi|^{\beta} \mathcal{F}(v)(\xi)\right)(x)
$$

for every $v \in D\left((-\Delta)^{\beta / 2}\right)=H^{\beta}\left(\mathbb{R}^{N}\right)$, where $H^{\beta}\left(\mathbb{R}^{N}\right)$ is the homogeneous Sobolev space of order $\beta$ defined by

$$
\begin{array}{ll}
H^{\beta}\left(\mathbb{R}^{N}\right)=\left\{u \in \mathcal{S}^{\prime} ;(-\Delta)^{\beta / 2} u \in L^{2}\left(\mathbb{R}^{N}\right)\right\} & \text { if } \beta \notin \mathbb{N}, \\
H^{\beta}\left(\mathbb{R}^{N}\right)=\left\{u \in L^{2}\left(\mathbb{R}^{N}\right) ;(-\Delta)^{\beta / 2} u \in L^{2}\left(\mathbb{R}^{N}\right)\right\} & \text { if } \beta \in \mathbb{N},
\end{array}
$$

Received August 10, 2010.

2000 Mathematics Subject Classification. Primary 26A33, 35B33, 35K55; Secondary 74G25, 74H35, $74 \mathrm{G} 40$.

Key words and phrases. Parabolic equation, fractional Laplacian, Riemann-Liouville fractional integrals and derivatives, local existence, critical exponent, blow-up rate.

E-mail address: ahmad.fino01@gmail.com

E-mail address: mokhtar.kirane@univ-lr.fr 
where $\mathcal{S}^{\prime}$ is the space of Schwartz distributions, $\mathcal{F}$ stands for the Fourier transform and $\mathcal{F}^{-1}$ for its inverse, $\Gamma$ is the Euler gamma function, and $C_{0}\left(\mathbb{R}^{N}\right)$ denotes the space of all continuous functions tending to zero at infinity.

When equation (1.1) is considered with a nonlinearity of the form $u^{p}$, it reads

$$
u_{t}+(-\Delta)^{\beta / 2} u=u^{p} .
$$

This equation has been considered by Nagasawa and Sirao [24, Kobayashi [20], Guedda and Kirane [14, Kirane and Qafsaoui [19, Eidelman and Kochubei [10, and by Fino and Karch [12].

The fractional Laplacian $(-\Delta)^{\beta / 2}$ is related to Lévy flights in physics. Many observations and experiments related to Lévy flights (super-diffusion), e.g., collective slip diffusion on solid surfaces, quantum optics, or Richardson turbulent diffusion have been performed in recent years. The symmetric $\beta$-stable processes $(\beta \in(0,2))$ are the basic characteristics for a class of jumping Lévy's processes. Compared with the continuous Brownian motion $(\beta=2)$, symmetric $\beta$-stable processes have infinite jumps in an arbitrary time interval. The large jumps of these processes make their variances and expectations infinite according to $\beta \in(0,2)$ or $\beta \in(0,1]$, respectively (see [17]). Let us also mention that when $\beta=3 / 2$, the symmetric $\beta$-stable processes appear in the study of stellar dynamics (see [9]).

As a physical motivation, the problem (1.1) describes a diffusion in a super-diffusive medium coupled to a classically diffusive medium. The right-hand side of (1.1) might be interpreted as the effect of a classically diffusive medium that is nonlinearly linked to a super-diffusive medium. Such a link might come in the form of a porous material with reactive properties that is partially insulated by contact with a classically diffusive material (thanks to Olmstead [25]). For more information, see the recent paper of Roberts and Olmstead [26].

Our article is motivated mathematically by the recent and very interesting paper [8] which deals with the global existence and blow-up for the parabolic equation with nonlocal in time nonlinearity

$$
u_{t}-\Delta u=\int_{0}^{t}(t-s)^{-\gamma}|u|^{p-1} u(s) d s \quad x \in \mathbb{R}^{N}, t>0,
$$

where $0 \leq \gamma<1, p>1$ and $u_{0} \in C_{0}\left(\mathbb{R}^{N}\right)$, which is a particular case of (1.1); it corresponds to $\beta=2$.

If we set

$$
p_{\gamma}=1+\frac{2(2-\gamma)}{(N-2+2 \gamma)_{+}}
$$

and

$$
p_{*}=\max \left\{\frac{1}{\gamma}, p_{\gamma}\right\} \in(0,+\infty],
$$

where $(\cdot)_{+}$is the positive part, they proved that

(i) If $\gamma \neq 0, p \leq p_{*}$, and $u_{0} \geq 0, u_{0} \neq \equiv 0$, then $u$ blows up in finite time.

(ii) If $\gamma \neq 0, p>p_{*}$, and $u_{0} \in L^{q_{s c}}\left(\mathbb{R}^{N}\right)$ (where $q_{s c}=N(p-1) /(4-2 \gamma)$ ) with $\left\|u_{0}\right\|_{L^{q_{s c}}}$ sufficiently small, then $u$ exists globally. 
If $\gamma=0$, then all nontrivial positive solutions blow up as proved by Souplet in 29]. The study in 8 . reveals the surprising fact that for equation (1.2) the critical exponent in Fujita's sense $p_{*}$ is not the one predicted by scaling.

This can be explained by the fact that their equation can be formally converted into

$$
D_{0 \mid t}^{\alpha} u_{t}-D_{0 \mid t}^{\alpha} \Delta u=|u|^{p-1} u,
$$

where $D_{0 \mid t}^{\alpha}$ is the left-hand side of the Riemann-Liouville fractional derivative of order $\alpha \in(0,1)$ defined in (2.8) below (we have set in (1.3), $\alpha=1-\gamma \in(0,1)$ ).

Equation (1.3) is a pseudo-parabolic equation and, as is well known, scaling is efficient for detecting the Fujita exponent only for equations of parabolic type.

Needless to say that the equation considered in [8] is a genuine extension of the one considered by Fujita in his pioneering work [13].

In this article, concerning blowing-up solutions, we present a different proof from the one presented in [8, and for the more general equation (1.1). Our proof is more versatile and can be applied to more nonlinear equations (see the Remarks in Section 4).

Our analysis is based on the observation that the nonlinear differential equation (1.1) can be written in the form

$$
u_{t}+(-\Delta)^{\beta / 2} u=J_{0 \mid t}^{\alpha}\left(|u|^{p-1} u\right),
$$

where $\alpha:=1-\gamma \in(0,1)$ and $J_{0 \mid t}^{\alpha}$ is the Riemann-Liouville fractional integral defined in (2.10).

We will show the following:

(1) For $u_{0} \in C_{0}\left(\mathbb{R}^{N}\right), u_{0} \geq 0, u_{0} \not \equiv 0$, if

$$
p \leq 1+\frac{\beta(2-\gamma)}{(N-\beta+\beta \gamma)_{+}} \quad \text { or } \quad p<\frac{1}{\gamma},
$$

then all solutions of problem (1.1) blow up in finite time.

(2) For $u_{0} \in C_{0}\left(\mathbb{R}^{N}\right) \cap L^{p_{s c}}\left(\mathbb{R}^{N}\right)$, where $p_{s c}:=N(p-1) / \beta(2-\gamma)$, if

$$
p>\max \left\{1+\frac{\beta(2-\gamma)}{(N-\beta+\beta \gamma)_{+}} ; \frac{1}{\gamma}\right\},
$$

and $\left\|u_{0}\right\|_{L^{p_{s c}}}$ is sufficiently small, then $u$ exists globally.

The method used to prove the blow-up theorem is the test function method of Mitidieri and Pohozaev [23, Kirane et al. [14, 18]; it was also used by Baras and Kersner 2] for the study of the necessary conditions for the local existence.

Furthermore, in the case $\beta=2$, we derive the blow-up rate estimates for the parabolic equation (1.1). We shall prove that, if $u_{0} \in C_{0}\left(\mathbb{R}^{N}\right) \cap L^{2}\left(\mathbb{R}^{N}\right), u_{0} \geq 0, u_{0} \not \equiv 0$ and if $u$ is the blowing-up solution of (1.1) at the finite time $T^{*}>0$, then there are constants $c, C>0$ such that $c\left(T^{*}-t\right)^{-\alpha_{1}} \leq \sup _{\mathbb{R}^{N}} u(\cdot, t) \leq C\left(T^{*}-t\right)^{-\alpha_{1}}$ for $1<p \leq 1+$ $2(2-\gamma) /(N-2+2 \gamma)_{+}$or $1<p<1 / \gamma$ and all $t \in\left(0, T^{*}\right)$, where $\alpha_{1}:=(2-\gamma) /(p-1)$. We use a scaling argument to reduce the problems of the blow-up rate to Fujita-type theorems (it is similar to the blow-up analysis in elliptic problems to reduce the problems of a priori bounds to Liouville-type theorems). As far as we know, this method was first applied to parabolic problems by $\mathrm{Hu}[15$, and then was used in various parabolic equations and 
systems (see [7, 11]). We notice that in the limiting case when $\gamma \rightarrow 0$, we obtain the constant rate $2 /(p-1)$ found by P. Souplet 30.

For more information, we refer the reader to the excellent paper of Andreucci and Tedeev [1] for the blow-up rate by an alternative method

The organization of this paper is as follows. In Section 2, we present some definitions and properties. In Section 3, we derive the local existence of solutions for the parabolic equation (1.1). Section 4 contains the blow-up result of solutions for (1.1). Section 5 is dedicated to the blow-up rate of blowing-up solutions. Global existence is studied in Section 6. Finally, a necessary condition for local existence and a necessary condition for global existence are given in Section 7 .

2. Preliminaries. In this section, we present some definitions and results concerning the fractional Laplacian, fractional integrals, and fractional derivatives that will be used hereafter.

First, if we take the usual linear fractional diffusion equation

$$
u_{t}+(-\Delta)^{\beta / 2} u=0, \quad \beta \in(0,2], x \in \mathbb{R}^{N}, t>0,
$$

then, its fundamental solution $S_{\beta}$ can be represented via the Fourier transform by

$$
S_{\beta}(t)(x):=S_{\beta}(x, t)=\frac{1}{(2 \pi)^{N / 2}} \int_{\mathbb{R}^{N}} e^{i x \cdot \xi-t|\xi|^{\beta}} d \xi .
$$

It is well known that this function satisfies

$$
S_{\beta}(1) \in L^{\infty}\left(\mathbb{R}^{N}\right) \cap L^{1}\left(\mathbb{R}^{N}\right), \quad S_{\beta}(x, t) \geq 0, \quad \int_{\mathbb{R}^{N}} S_{\beta}(x, t) d x=1,
$$

for all $x \in \mathbb{R}^{N}$ and $t>0$. Hence, using Young's inequality for the convolution and the following self-similar form $S_{\beta}(x, t)=t^{-N / \beta} S_{\beta}\left(x t^{-1 / \beta}, 1\right)$, we have

$$
\left\|S_{\beta}(t) * v\right\|_{q} \leq C t^{-\frac{N}{\beta}\left(\frac{1}{r}-\frac{1}{q}\right)}\|v\|_{r}
$$

for all $v \in L^{r}\left(\mathbb{R}^{N}\right)$ and all $1 \leq r \leq q \leq \infty, t>0$.

Moreover, as $(-\Delta)^{\beta / 2}$ is a self-adjoint operator with $D\left((-\Delta)^{\beta / 2}\right)=H^{\beta}\left(\mathbb{R}^{N}\right)$, we have

$$
\int_{\mathbb{R}^{N}} u(x)(-\Delta)^{\beta / 2} v(x) d x=\int_{\mathbb{R}^{N}} v(x)(-\Delta)^{\beta / 2} u(x) d x,
$$

for all $u, v \in H^{\beta}\left(\mathbb{R}^{N}\right)$.

We denote by $\Delta_{D}^{\beta / 2}$ the fractional Laplacian in an open bounded domain $\Omega$ with homogeneous Dirichlet boundary condition. We have the following facts:

If $\lambda_{k}(k=1, \ldots,+\infty)$ are the eigenvalues for the Laplacian operator in $L^{2}(\Omega)$ with homogeneous Dirichlet boundary condition and $\varphi_{k}$ its corresponding eigenfunction, then

$$
\begin{cases}\Delta_{D}^{\beta / 2} \varphi_{k}=\lambda_{k}^{\beta / 2} \varphi_{k} & \text { in } \Omega, \\ \varphi_{k}=0 & \text { on } \partial \Omega,\end{cases}
$$

and

$$
D\left(\Delta_{D}^{\beta / 2}\right)=\left\{u \in L^{2}(\Omega) \text { s.t. }\left.u\right|_{\partial \Omega}=0 ;\left\|\Delta_{D}^{\beta / 2} u\right\|_{L^{2}(\Omega)}:=\sum_{k=1}^{k=+\infty}\left|\lambda_{k}^{\beta / 2}\left\langle u, \varphi_{k}\right\rangle\right|^{2}<+\infty\right\} .
$$


So, for $u \in D\left(\Delta_{D}^{\beta / 2}\right)$, we have

$$
\Delta_{D}^{\beta / 2} u=\sum_{k=1}^{k=+\infty} \lambda_{k}^{\beta / 2}<u, \varphi_{k}>\varphi_{k} .
$$

The following integration by parts formula

$$
\int_{\Omega} u(x) \Delta_{D}^{\beta / 2} v(x) d x=\int_{\Omega} v(x) \Delta_{D}^{\beta / 2} u(x) d x,
$$

holds for all $u, v \in D\left(\Delta_{D}^{\beta / 2}\right)$.

Next, if $A C[0, T]$ is the space of all functions which are absolutely continuous on $[0, T]$ with $0<T<\infty$, then, for $f \in A C[0, T]$, the left-hand side and right-hand side of the Riemann-Liouville fractional derivatives $D_{0 \mid t}^{\alpha} f(t)$ and $D_{t \mid T}^{\alpha} f(t)$ of order $\alpha \in(0,1)$ are defined by

$$
\begin{aligned}
D_{0 \mid t}^{\alpha} f(t) & :=D J_{0 \mid t}^{1-\alpha} f(t), \\
D_{t \mid T}^{\alpha} f(t) & :=-\frac{1}{\Gamma(1-\alpha)} D \int_{t}^{T}(s-t)^{-\alpha} f(s) d s,
\end{aligned}
$$

(see [17]) for all $t \in[0, T]$, where $D:=d / d t$ is the usual time derivative and

$$
J_{0 \mid t}^{\alpha} f(t):=\frac{1}{\Gamma(\alpha)} \int_{0}^{t}(t-s)^{\alpha-1} f(s) d s
$$

is the Riemann-Liouville fractional integral defined for all $f \in L^{q}(0, T)(1 \leq q \leq \infty)$.

Now, for every $f, g \in C([0, T])$, such that $D_{0 \mid t}^{\alpha} f(t), D_{t \mid T}^{\alpha} g(t)$ exist and are continuous, for all $t \in[0, T], 0<\alpha<1$, we have the formula of integration by parts (see [28, (2.64) p. 46])

$$
\int_{0}^{T}\left(D_{0 \mid t}^{\alpha} f\right)(t) g(t) d t=\int_{0}^{T} f(t)\left(D_{t \mid T}^{\alpha} g\right)(t) d t .
$$

Note also that, for any $f \in A C^{2}[0, T]$, we have (see [17, (2.2.30)])

$$
-D . D_{t \mid T}^{\alpha} f=D_{t \mid T}^{1+\alpha} f,
$$

where

$$
A C^{2}[0, T]:=\{f:[0, T] \rightarrow \mathbb{R} \text { such that } D f \in A C[0, T]\} .
$$

Moreover, for all $1 \leq q \leq \infty$, the following equality (see [17, Lemma 2.4 p. 74])

$$
D_{0 \mid t}^{\alpha} J_{0 \mid t}^{\alpha}=I d_{L^{q}(0, T)}
$$

holds almost everywhere on $[0, T]$.

Later on, we will use the following results.

- If $w_{1}(t)=(1-t / T)_{+}^{\sigma}, t \geq 0, T>0, \sigma \gg 1$, then

$$
\begin{aligned}
D_{t \mid T}^{\alpha} w_{1}(t) & =\frac{(1-\alpha+\sigma) \Gamma(\sigma+1)}{\Gamma(2-\alpha+\sigma)} T^{-\alpha}\left(1-\frac{t}{T}\right)_{+}^{\sigma-\alpha} \\
D_{t \mid T}^{\alpha+1} w_{1}(t) & =\frac{(1-\alpha+\sigma)(\sigma-\alpha) \Gamma(\sigma+1)}{\Gamma(2-\alpha+\sigma)} T^{-(\alpha+1)}\left(1-\frac{t}{T}\right)_{+}^{\sigma-\alpha-1}
\end{aligned}
$$


for all $\alpha \in(0,1)$; so

$$
\left(D_{t \mid T}^{\alpha} w_{1}\right)(T)=0, \quad\left(D_{t \mid T}^{\alpha} w_{1}\right)(0)=C T^{-\alpha},
$$

where $C=(1-\alpha+\sigma) \Gamma(\sigma+1) / \Gamma(2-\alpha+\sigma)$.

- If $w_{2}(t)=\left(1-t^{2} / T^{2}\right)_{+}^{\ell}, T>0, \ell \gg 1$, we have

$$
\begin{aligned}
D_{t \mid T}^{\alpha} w_{2}(t) & =\frac{T^{-\alpha}}{\Gamma(1-\alpha)} \sum_{k=0}^{\ell} C_{1}(\ell, k, \alpha)\left(1-\frac{t}{T}\right)^{\ell+k-\alpha}, \\
D_{t \mid T}^{1+\alpha} w_{2}(t) & =\frac{T^{-\alpha-1}}{\Gamma(1-\alpha)} \sum_{k=0}^{\ell} C_{2}(\ell, k, \alpha)\left(1-\frac{t}{T}\right)^{\ell+k-\alpha-1},
\end{aligned}
$$

for all $-T \leq t \leq T, \alpha \in(0,1)$, where

$$
\left\{\begin{array}{l}
C_{1}(\ell, k, \alpha):=c_{\ell}^{k}(1-\alpha+\ell+k) 2^{\ell-k}(-1)^{k} \frac{\Gamma(k+\ell+1) \Gamma(1-\alpha)}{\Gamma(k+\ell+2-\alpha)} \\
C_{2}(\ell, k, \alpha):=(\ell+k-\alpha) C_{1}(\ell, k, \alpha) \\
c_{\ell}^{k}:=\frac{\ell !}{(\ell-k) ! k !}
\end{array}\right.
$$

so

$$
\left(D_{t \mid T}^{\alpha} w_{2}\right)(T)=0, \quad\left(D_{t \mid T}^{\alpha} w_{2}\right)(-T)=C_{3}(\ell, k, \alpha) T^{-\alpha}
$$

where

$$
C_{3}(\ell, k, \alpha):=\frac{2^{2 \ell-\alpha}(-1)^{\ell}}{\Gamma(1-\alpha)} \sum_{k=0}^{\ell} c_{\ell}^{k}(1-\alpha+\ell+k) \frac{\Gamma(k+\ell+1) \Gamma(1-\alpha)}{\Gamma(k+\ell+2-\alpha)} .
$$

3. Local existence. This section is dedicated to proving the local existence and uniqueness of mild solutions to the problem (1.1). Let $T(t):=e^{-t(-\Delta)^{\beta / 2}}$. As $(-\Delta)^{\beta / 2}$ is a positive definite self-adjoint operator in $L^{2}\left(\mathbb{R}^{N}\right), T(t)$ is a strongly continuous semigroup on $L^{2}\left(\mathbb{R}^{N}\right)$ generated by the fractional power $-(-\Delta)^{\beta / 2}$ (see Yosida [31]). It holds that $T(t) v=S_{\beta}(t) * v$, where $S_{\beta}$ is given by (2.2) and $u * v$ is the convolution of $u$ and $v$. We start by giving the

Definition 3.1 (Mild solution). Let $u_{0} \in C_{0}\left(\mathbb{R}^{N}\right), 0<\beta \leq 2, p>1$, and $T>0$. We say that $u \in C\left([0, T], C_{0}\left(\mathbb{R}^{N}\right)\right)$ is a mild solution of problem (1.1) if $u$ satisfies the integral equation

$$
u(t)=T(t) u_{0}+\int_{0}^{t} T(t-s) J_{0 \mid s}^{\alpha}\left(|u|^{p-1} u\right) d s, \quad t \in[0, T] .
$$

Theorem 3.2 (Local existence). Given $u_{0} \in C_{0}\left(\mathbb{R}^{N}\right)$ and $p>1$, there exist a maximal time $T_{\max }>0$ and a unique mild solution $u \in C\left(\left[0, T_{\max }\right), C_{0}\left(\mathbb{R}^{N}\right)\right)$ to problem (1.1). Furthermore, either $T_{\max }=\infty$ or else $T_{\max }<\infty$ and $\|u\|_{L^{\infty}\left((0, t) \times \mathbb{R}^{N}\right)} \rightarrow \infty$ as $t \rightarrow T_{\max }$. In addition, if $u_{0} \geq 0, u_{0} \not \equiv 0$, then $u(t)>0$ for all $0<t<T_{\max }$. Moreover, if $u_{0} \in L^{r}\left(\mathbb{R}^{N}\right)$, for $1 \leq r<\infty$, then $u \in C\left(\left[0, T_{\max }\right), L^{r}\left(\mathbb{R}^{N}\right)\right)$.

Proof. For arbitrary $T>0$, we define the Banach space

$$
E_{T}:=\left\{u \in L^{\infty}\left((0, T), C_{0}\left(\mathbb{R}^{N}\right)\right) ;\|u\|_{1} \leq 2\left\|u_{0}\right\|_{L^{\infty}}\right\},
$$


where $\|\cdot\|_{1}:=\|\cdot\|_{L^{\infty}\left((0, T), L^{\infty}\left(\mathbb{R}^{N}\right)\right)}$. Next, for every $u \in E_{T}$, we define

$$
\Psi(u):=T(t) u_{0}+\int_{0}^{t} T(t-s) J_{0 \mid s}^{\alpha}\left(|u|^{p-1} u\right) d s .
$$

We prove the local existence by the Banach fixed point theorem.

$\bullet \Psi: \mathbf{E}_{\mathbf{T}} \rightarrow \mathbf{E}_{\mathbf{T}}$. Let $u \in E_{T}$, using (2.4), we obtain with $\|\cdot\|_{\infty}:=\|\cdot\|_{L^{\infty}\left(\mathbb{R}^{N}\right)}$,

$$
\begin{aligned}
\|\Psi(u)\|_{1} & \leq\left\|u_{0}\right\|_{\infty}+\frac{1}{\Gamma(1-\gamma)}\left\|\int_{0}^{t} \int_{0}^{s}(s-\sigma)^{-\gamma}\right\| u(\sigma)\left\|_{\infty}^{p} d \sigma d s\right\|_{L^{\infty}(0, T)} \\
& =\left\|u_{0}\right\|_{\infty}+\frac{1}{\Gamma(1-\gamma)}\left\|\int_{0}^{t} \int_{\sigma}^{t}(s-\sigma)^{-\gamma}\right\| u(\sigma)\left\|_{\infty}^{p} d s d \sigma\right\|_{L^{\infty}(0, T)} \\
& \leq\left\|u_{0}\right\|_{\infty}+\frac{T^{2-\gamma}}{(1-\gamma)(2-\gamma) \Gamma(1-\gamma)}\|u\|_{1}^{p} \\
& \leq\left\|u_{0}\right\|_{\infty}+\frac{T^{2-\gamma} 2^{p}\left\|u_{0}\right\|_{L^{\infty}}^{p-1}}{\Gamma(3-\gamma)}\left\|u_{0}\right\|_{\infty} .
\end{aligned}
$$

Now, if we choose $T$ small enough such that

$$
\frac{T^{2-\gamma} 2^{p}\left\|u_{0}\right\|_{\infty}^{p-1}}{\Gamma(3-\gamma)} \leq 1
$$

we conclude that $\|\Psi(u)\|_{1} \leq 2\left\|u_{0}\right\|_{\infty}$, and then $\Psi(u) \in E_{T}$.

- $\Psi$ is a contraction. For $u, v \in E_{T}$, taking account of (2.4), we have

$$
\begin{aligned}
\|\Psi(u)-\Psi(v)\|_{1} & \leq \frac{1}{\Gamma(1-\gamma)}\left\|\int_{0}^{t} \int_{0}^{s}(s-\sigma)^{-\gamma}\right\||u|^{p-1} u(\sigma)-|v|^{p-1} v(\sigma)\left\|_{\infty} d \sigma d s\right\|_{L^{\infty}(0, T)} \\
& =\left.\frac{1}{\Gamma(1-\gamma)}\left\|\int_{0}^{t} \int_{\sigma}^{t}(s-\sigma)^{-\gamma}\right\| u\right|^{p-1} u(\sigma)-|v|^{p-1} v(\sigma)\left\|_{\infty} d s d \sigma\right\|_{L^{\infty}(0, T)} \\
& \leq \frac{T^{2-\gamma}}{\Gamma(3-\gamma)}\left\|\left.u\right|^{p-1} u-|v|^{p-1} v\right\|_{1} \\
& \leq \frac{C(p) 2^{p}\left\|u_{0}\right\|_{\infty}^{p-1} T^{2-\gamma}}{\Gamma(3-\gamma)}\|u-v\|_{1} \\
& \leq \frac{1}{2}\|u-v\|_{1},
\end{aligned}
$$

thanks to the following inequality

$$
\left.|| u\right|^{p-1} u-|v|^{p-1} v|\leq C(p)| u-v \mid\left(|u|^{p-1}+|v|^{p-1}\right)
$$

$T$ is chosen such that

$$
\frac{T^{2-\gamma} 2^{p}\left\|u_{0}\right\|_{\infty}^{p-1} \max (2 C(p), 1)}{\Gamma(3-\gamma)} \leq 1 .
$$

Then, by the Banach fixed point theorem, there exists a mild solution $u \in \Pi_{T}$, where $\Pi_{T}:=L^{\infty}\left((0, T), C_{0}\left(\mathbb{R}^{N}\right)\right)$, to the problem (1.1). 
- Uniqueness. If $u, v$ are two mild solutions in $E_{T}$ for some $T>0$, using (2.4) and (3.3), we obtain

$$
\begin{aligned}
\|u(t)-v(t)\|_{\infty} & \leq \frac{C(p) 2^{p}\left\|u_{0}\right\|_{\infty}^{p-1}}{\Gamma(1-\gamma)} \int_{0}^{t} \int_{0}^{s}(s-\sigma)^{-\gamma}\|u(\sigma)-v(\sigma)\|_{\infty} d \sigma d s \\
& =\frac{C(p) 2^{p}\left\|u_{0}\right\|_{\infty}^{p-1}}{\Gamma(1-\gamma)} \int_{0}^{t} \int_{\sigma}^{t}(s-\sigma)^{-\gamma}\|u(\sigma)-v(\sigma)\|_{\infty} d s d \sigma \\
& =\frac{C(p) 2^{p}\left\|u_{0}\right\|_{\infty}^{p-1}}{\Gamma(2-\gamma)} \int_{0}^{t}(t-\sigma)^{1-\gamma}\|u(\sigma)-v(\sigma)\|_{\infty} d \sigma .
\end{aligned}
$$

So the uniqueness follows from Gronwall's inequality (cf. [6]).

Next, using the uniqueness of solutions, we conclude the existence of a solution on a maximal interval $\left[0, T_{\max }\right)$ where

$$
T_{\max }:=\sup \left\{T>0 \text {; there exist a mild solution } u \in \Pi_{T} \text { to }(1.1)\right\} \leq+\infty .
$$

Note that, using the continuity of the semigroup $T(t)$, we can easily conclude that

$$
u \in C\left(\left[0, T_{\max }\right), C_{0}\left(\mathbb{R}^{N}\right)\right) .
$$

Moreover, if $0 \leq t \leq t+\tau<T_{\max }$, using (3.1), we can write

$$
\begin{aligned}
u(t+\tau) & =T(\tau) u(t)+\frac{1}{\Gamma(1-\gamma)} \int_{0}^{\tau} T(\tau-s) \int_{0}^{s}(s-\sigma)^{-\gamma}|u|^{p-1} u(t+\sigma) d \sigma d s \\
& +\frac{1}{\Gamma(1-\gamma)} \int_{0}^{\tau} T(\tau-s) \int_{0}^{t}(t+s-\sigma)^{-\gamma}|u|^{p-1} u(\sigma) d \sigma d s .
\end{aligned}
$$

To prove that $\|u(t)\|_{L^{\infty}\left(\mathbb{R}^{N}\right)} \rightarrow \infty$ as $t \rightarrow T_{\max }$, whenever $T_{\max }<\infty$, we proceed by contradiction. Suppose that $u$ is a solution of (3.1) on some interval $[0, T)$ with $\|u\|_{L^{\infty}\left((0, T) \times \mathbb{R}^{N}\right)}<\infty$ and $T_{\max }<\infty$. Using the fact that the last term in (3.5) depends only on the values of $u$ in the interval $(0, t)$ and using again a fixed point argument, we conclude that $u$ can be extended to a solution on some interval $\left[0, T^{\prime}\right)$ with $T^{\prime}>T$. If we repeat this iteration, we obtain a contradiction with the fact that the maximal time $T_{\max }$ is finite.

- Positivity of solutions. If $u_{0} \geq 0$ and $u_{0} \not \equiv 0$, then we can construct a nonnegative solution on some interval $[0, T]$ by applying the fixed point argument in the set $E_{T}^{+}=$ $\left\{u \in E_{T} ; u \geq 0\right\}$. In particular, it follows from (3.1) that $u(t) \geq T(t) u_{0}>0$ on $(0, T]$. It is not difficult by uniqueness to deduce that $u$ stays positive on $\left(0, T_{\max }\right)$.

- Regularity. If $u_{0} \in L^{r}\left(\mathbb{R}^{N}\right) \cap C_{0}\left(\mathbb{R}^{N}\right)$, for $1 \leq r<\infty$, then by repeating the fixed point argument in the space

$$
E_{T, r}:=\left\{u \in L^{\infty}\left((0, T), C_{0}\left(\mathbb{R}^{N}\right) \cap L^{r}\left(\mathbb{R}^{N}\right)\right) ;\|u\|_{1} \leq 2\left\|u_{0}\right\|_{L^{\infty}},\|u\|_{\infty, r} \leq 2\left\|u_{0}\right\|_{L^{r}}\right\},
$$

instead of $E_{T}$, where $\|\cdot\|_{\infty, r}:=\|\cdot\|_{L^{\infty}\left((0, T), L^{r}\left(\mathbb{R}^{N}\right)\right)}$, and by estimating $\left\|u^{p}\right\|_{L^{r}\left(\mathbb{R}^{N}\right)}$ by $\|u\|_{L^{\infty}\left(\mathbb{R}^{N}\right)}^{p-1}\|u\|_{L^{r}\left(\mathbb{R}^{N}\right)}$ in the contraction mapping argument, using (2.4), we obtain a unique solution in $E_{T, r}$. We conclude then that

$$
u \in C\left(\left[0, T_{\max }\right), C_{0}\left(\mathbb{R}^{N}\right) \cap L^{r}\left(\mathbb{R}^{N}\right)\right) .
$$

We say that $u$ is a global solution if $T_{\max }=\infty$; when $T_{\max }<\infty, u$ is said to blow up in a finite time, and in this case we have $\|u(\cdot, t)\|_{L^{\infty}\left(\mathbb{R}^{N}\right)} \rightarrow \infty$ as $t \rightarrow T_{\max }$. 
REMARK. We note that classical or strong solutions do not exist due to the singularity in time in the nonlinear term.

4. Blow-up of solutions. Now, we want to derive a blow-up result for equation (1.1). Our argument uses weak solutions.

Definition 4.1 (Weak solution). Let $u_{0} \in L_{\text {Loc }}^{\infty}\left(\mathbb{R}^{N}\right), 0<\beta \leq 2$ and $T>0$. We say that $u$ is a weak solution of the problem (1.1) if $u \in L^{p}\left((0, T), L_{\mathrm{Loc}}^{\infty}\left(\mathbb{R}^{N}\right)\right)$ and verifies the equation

$$
\begin{aligned}
\int_{\mathbb{R}^{N}} u_{0}(x) \varphi(x, 0)+\int_{0}^{T} \int_{\mathbb{R}^{N}} J_{0 \mid t}^{\alpha}\left(|u|^{p-1} u\right)(x, t) \varphi(x, t) & =\int_{0}^{T} \int_{\mathbb{R}^{N}} u(x, t)(-\Delta)^{\beta / 2} \varphi(x, t) \\
& -\int_{0}^{T} \int_{\mathbb{R}^{N}} u(x, t) \varphi_{t}(x, t), \quad \text { (4.1) }
\end{aligned}
$$

for all compactly supported $\varphi \in C^{1}\left([0, T], H^{\beta}\left(\mathbb{R}^{N}\right)\right)$ such that $\varphi(\cdot, T)=0$, where $\alpha:=$ $1-\gamma \in(0,1)$.

Lemma 4.2. Consider $u_{0} \in C_{0}\left(\mathbb{R}^{N}\right)$, and let $u \in C\left([0, T], C_{0}\left(\mathbb{R}^{N}\right)\right)$ be a mild solution of (1.1), then $u$ is a weak solution of (1.1), for all $0<\beta \leq 2$ and all $T>0$.

Proof. Let $T>0,0<\beta \leq 2, u_{0} \in C_{0}\left(\mathbb{R}^{N}\right)$, and let $u \in C\left([0, T], C_{0}\left(\mathbb{R}^{N}\right)\right)$ be a solution of (3.1). Given $\varphi \in C^{1}\left([0, T], H^{\beta}\left(\mathbb{R}^{N}\right)\right)$ such that $\operatorname{supp} \varphi$ is compact with $\varphi(\cdot, T)=0$, then after multiplying (3.1) by $\varphi$ and integrating over $\mathbb{R}^{N}$, we have

$$
\begin{aligned}
\int_{\mathbb{R}^{N}} u(x, t) \varphi(x, t)= & \int_{\mathbb{R}^{N}} T(t) u_{0}(x) \varphi(x, t) \\
& +\int_{\mathbb{R}^{N}}\left(\int_{0}^{t} T(t-s) J_{0 \mid s}^{\alpha}\left(|u|^{p-1} u\right)(x, t) d s\right) \varphi(x, t) .
\end{aligned}
$$

We differentiate to obtain

$$
\begin{aligned}
\frac{d}{d t} \int_{\mathbb{R}^{N}} u(x, t) \varphi(x, t) & =\int_{\mathbb{R}^{N}} \frac{d}{d t}\left(T(t) u_{0}(x) \varphi(x, t)\right) \\
& +\int_{\mathbb{R}^{N}} \frac{d}{d t} \int_{0}^{t} T(t-s) J_{0 \mid s}^{\alpha}\left(|u|^{p-1} u\right)(x, s) d s \varphi(x, t) .
\end{aligned}
$$

Now, using (2.5) and a property of the semigroup $T(t)$ ([6, Chapter 3]), we have

$$
\begin{aligned}
\int_{\mathbb{R}^{N}} \frac{d}{d t}\left(T(t) u_{0}(x) \varphi(x, t)\right) & =\int_{\mathbb{R}^{N}} A\left(T(t) u_{0}(x)\right) \varphi(x, t)+\int_{\mathbb{R}^{N}} T(t) u_{0}(x) \varphi_{t}(x, t) \\
& =\int_{\mathbb{R}^{N}} T(t) u_{0}(x) A \varphi(x, t)+\int_{\mathbb{R}^{N}} T(t) u_{0}(x) \varphi_{t}(x, t)
\end{aligned}
$$


and

$$
\begin{aligned}
\int_{\mathbb{R}^{N}} & \frac{d}{d t} \int_{0}^{t} T(t-s) f(x, s) d s \varphi(x, t) \\
= & \int_{\mathbb{R}^{N}} f(x, t) \varphi(x, t)+\int_{\mathbb{R}^{N}} \int_{0}^{t} A(T(t-s) f(x, s)) d s \varphi(x, t) \\
& +\int_{\mathbb{R}^{N}} \int_{0}^{t} T(t-s) f(x, s) d s \varphi_{t}(x, t) \\
= & \int_{\mathbb{R}^{N}} f(x, t) \varphi(x, t)+\int_{\mathbb{R}^{N}} \int_{0}^{t} T(t-s) f(x, s) d s A \varphi(x, t) \\
& +\int_{\mathbb{R}^{N}} \int_{0}^{t} T(t-s) f(x, s) d s \varphi_{t}(x, t),
\end{aligned}
$$

where $f:=J_{0 \mid t}^{\alpha}\left(|u|^{p-1} u\right) \in C\left([0, T] ; L^{2}\left(\mathbb{R}^{N}\right)\right)$.

Thus, using (3.1), (4.3), and (4.4), we conclude that (4.2) implies

$$
\frac{d}{d t} \int_{\mathbb{R}^{N}} u(x, t) \varphi(x, t)=\int_{\mathbb{R}^{N}} u(x, t) A \varphi(x, t)+\int_{\mathbb{R}^{N}} u(x, t) \varphi_{t}(x, t)+\int_{\mathbb{R}^{N}} f(x, t) \varphi(x, t) .
$$

We conclude by integrating in time over $[0, T]$ and using the fact that $\varphi(\cdot, T)=0$.

Theorem 4.3. Let $u_{0} \in C_{0}\left(\mathbb{R}^{N}\right)$ be such that $u_{0} \geq 0$ and $u_{0} \not \equiv 0$. If

$$
p \leq 1+\frac{\beta(2-\gamma)}{(N-\beta+\beta \gamma)_{+}}:=p^{*} \quad \text { or } \quad p<\frac{1}{\gamma},
$$

for all $\beta \in(0,2]$, then the mild solution to (1.1) blows up in a finite time.

Note that in the case where $p=p^{*}$ and $\beta \in(0,2)$, we take $p>N /(N-\beta)$ with $N>\beta$.

Proof. The proof is by contradiction. Suppose that $u$ is a global mild solution to (1.1). Then $u$ is a solution of (1.1) in $C\left([0, T], C_{0}\left(\mathbb{R}^{N}\right)\right)$ for all $T \gg 1$ such that $u(t)>0$ for all $t \in[0, T]$.

Then, using Lemma 4.2, we have

$$
\begin{aligned}
\int_{\mathbb{R}^{N}} u_{0}(x) \varphi(x, 0)+\int_{0}^{T} \int_{\mathbb{R}^{N}} J_{0 \mid t}^{\alpha}\left(u^{p}\right)(x, t) \varphi(x, t) & =\int_{0}^{T} \int_{\mathbb{R}^{N}} u(x, t)(-\Delta)^{\beta / 2} \varphi(x, t) \\
& -\int_{0}^{T} \int_{\mathbb{R}^{N}} u(x, t) \varphi_{t}(x, t),
\end{aligned}
$$

for all test functions $\varphi \in C^{1}\left([0, T], H^{\beta}\left(\mathbb{R}^{N}\right)\right)$ such that $\operatorname{supp} \varphi$ is compact with $\varphi(\cdot, T)=$ 0 , where $\alpha:=1-\gamma \in(0,1)$.

Now we take $\varphi(x, t)=D_{t \mid T}^{\alpha}(\tilde{\varphi}(x, t)):=D_{t \mid T}^{\alpha}\left(\left(\varphi_{1}(x)\right)^{\ell} \varphi_{2}(t)\right)$ with $\varphi_{1}(x):=\Phi\left(|x| / T^{1 / \beta}\right)$, $\varphi_{2}(t):=(1-t / T)_{+}^{\eta}$, where $\ell \geq p /(p-1), \eta \geq \max \{(\alpha p+1) /(p-1) ; \alpha+1\}$, and $\Phi$ a smooth nonnegative nonincreasing function such that

$$
\Phi(r)= \begin{cases}1 & \text { if } 0 \leq r \leq 1, \\ 0 & \text { if } r \geq 2,\end{cases}
$$


$0 \leq \Phi \leq 1,\left|\Phi^{\prime}(r)\right| \leq C_{1} / r$, for all $r>0$. Using (2.16), we then obtain

$$
\begin{aligned}
& \int_{\Omega} u_{0}(x) D_{t \mid T}^{\alpha} \tilde{\varphi}(x, 0)+\int_{\Omega_{T}} J_{0 \mid t}^{\alpha}\left(u^{p}\right)(x, t) D_{t \mid T}^{\alpha} \tilde{\varphi}(x, t) \\
& =\int_{0}^{T} \int_{\mathbb{R}^{N}} u(x, t)(-\Delta)^{\beta / 2} D_{t \mid T}^{\alpha} \tilde{\varphi}(x, t)-\int_{\Omega_{T}} u(x, t) D D_{t \mid T}^{\alpha} \tilde{\varphi}(x, t),
\end{aligned}
$$

where

$\Omega_{T}:=[0, T] \times \Omega$ for $\Omega=\left\{x \in \mathbb{R}^{N} ;|x| \leq 2 T^{1 / \beta}\right\}, \quad \int_{\Omega}=\int_{\Omega} d x \quad$ and $\int_{\Omega_{T}}=\int_{\Omega_{T}} d x d t$.

Furthermore, using (2.11) and (2.16) in the left-hand side of (4.6) and (2.12) in the right-hand side, we obtain

$$
\begin{aligned}
& C T^{-\alpha} \int_{\Omega} u_{0}(x) \varphi_{1}^{\ell}(x)+\int_{\Omega_{T}} D_{0 \mid t}^{\alpha} J_{0 \mid t}^{\alpha}\left(u^{p}\right)(x, t) \tilde{\varphi}(x, t) \\
& =\int_{0}^{T} \int_{\mathbb{R}^{N}} u(x, t)(-\Delta)^{\beta / 2} D_{t \mid T}^{\alpha} \tilde{\varphi}(x, t)+\int_{\Omega_{T}} u(x, t) D_{t \mid T}^{1+\alpha} \tilde{\varphi}(x, t) .
\end{aligned}
$$

Moreover, using (2.13), we may write

$$
\begin{aligned}
& \int_{\Omega_{T}} u^{p}(x, t) \tilde{\varphi}(x, t)+C T^{-\alpha} \int_{\Omega} u_{0}(x) \varphi_{1}^{\ell}(x) \\
& =\int_{0}^{T} \int_{\mathbb{R}^{N}} u(x, t)(-\Delta)^{\beta / 2} \varphi_{1}^{\ell}(x) D_{t \mid T}^{\alpha} \varphi_{2}(t)+\int_{\Omega_{T}} u(x, t) D_{t \mid T}^{1+\alpha} \tilde{\varphi}(x, t) .
\end{aligned}
$$

So Ju's inequality $(-\Delta)^{\beta / 2}\left(\varphi_{1}^{\ell}\right) \leq \ell \varphi_{1}^{\ell-1}(-\Delta)^{\beta / 2}\left(\varphi_{1}\right)$ (see the Appendix) allows us to write

$$
\begin{aligned}
& \int_{\Omega_{T}} u^{p}(x, t) \tilde{\varphi}(x, t)+C T^{-\alpha} \int_{\Omega} u_{0}(x) \varphi_{1}^{\ell}(x) \\
& \leq C \int_{\Omega_{T}} u(x, t) \varphi_{1}^{\ell-1}(x)\left|(-\Delta)^{\beta / 2} \varphi_{1}(x) D_{t \mid T}^{\alpha} \varphi_{2}(t)\right| \\
& +\int_{\Omega_{T}} u(x, t) \varphi_{1}^{\ell}(x)\left|D_{t \mid T}^{1+\alpha} \varphi_{2}(t)\right| \\
& =C \int_{\Omega_{T}} u(x, t) \tilde{\varphi}^{1 / p} \tilde{\varphi}^{-1 / p} \varphi_{1}^{\ell-1}(x)\left|(-\Delta)^{\beta / 2} \varphi_{1}(x) D_{t \mid T}^{\alpha} \varphi_{2}(t)\right| \\
& +\int_{\Omega_{T}} u(x, t) \tilde{\varphi}^{1 / p} \tilde{\varphi}^{-1 / p} \varphi_{1}^{\ell}(x)\left|D_{t \mid T}^{1+\alpha} \varphi_{2}(t)\right| .
\end{aligned}
$$

Therefore, using Young's inequality

$$
a b \leq \frac{1}{2 p} a^{p}+\frac{2^{\tilde{p}-1}}{\tilde{p}} b^{\tilde{p}}, \quad \text { where } p \tilde{p}=p+\tilde{p}, \quad a>0, b>0, \quad p>1, \tilde{p}>1,
$$

with

$$
\left\{\begin{array}{l}
a=u(x, t) \tilde{\varphi}^{1 / p} \\
b=\tilde{\varphi}^{-1 / p} \varphi_{1}^{\ell-1}(x)\left|(-\Delta)^{\beta / 2} \varphi_{1}(x) D_{t \mid T}^{\alpha} \varphi_{2}(t)\right|
\end{array}\right.
$$


in the first integral of the right-hand side of (4.9), and with

$$
\left\{\begin{array}{l}
a=u(x, t) \tilde{\varphi}^{1 / p} \\
b=\tilde{\varphi}^{-1 / p} \varphi_{1}^{\ell}(x)\left|D_{t \mid T}^{1+\alpha} \varphi_{2}(t)\right|
\end{array}\right.
$$

in the second integral of the right-hand side of (4.9), we obtain

$$
\begin{aligned}
& \left(1-\frac{1}{p}\right) \int_{\Omega_{T}} u^{p}(x, t) \tilde{\varphi}(x, t) \\
& \leq C \int_{\Omega_{T}}\left(\varphi_{1}(x)\right)^{\ell-\tilde{p}}\left(\varphi_{2}(t)\right)^{-\frac{1}{p-1}}\left|\left(-\Delta_{x}\right)^{\beta / 2} \varphi_{1}(x) D_{t \mid T}^{\alpha} \varphi_{2}(t)\right|^{\tilde{p}} \\
& +C \int_{\Omega_{T}}\left(\varphi_{1}(x)\right)^{\ell}\left(\varphi_{2}(t)\right)^{-\frac{1}{p-1}}\left|D_{t \mid T}^{1+\alpha} \varphi_{2}(t)\right|^{\tilde{p}}
\end{aligned}
$$

as $u_{0} \geq 0$. At this stage, we introduce the scaled variables $\tau=T^{-1} t, \xi=T^{-1 / \beta} x$. We use formulas (2.14) and (2.15) in the right-hand side of (4.11) to obtain

$$
\int_{\Omega_{T}} u^{p}(x, t) \tilde{\varphi}(x, t) \leq C T^{-\delta}
$$

where $\delta:=(1+\alpha) \widetilde{p}-1-(N / \beta), C=C\left(\left|\Omega_{1}\right|,\left|\Omega_{2}\right|\right),\left(\left|\Omega_{i}\right|\right.$ stands for the measure of $\Omega_{i}$, for $i=1,2)$, with

$$
\Omega_{1}:=\left\{\xi \in \mathbb{R}^{N} ;|\xi| \leq 2\right\}, \quad \Omega_{2}:=\{\tau \geq 0 ; \tau \leq 1\} .
$$

Now, noting that, as

$$
p \leq p^{*} \quad \text { or } \quad p<\frac{1}{\gamma} \Longleftrightarrow \delta \geq 0 \quad \text { or } \quad p<\frac{1}{\gamma},
$$

we have to distinguish three cases:

- The case $p<p^{*}(\delta>0)$. We pass to the limit in (4.12), as $T$ goes to $\infty$. We get

$$
\lim _{T \rightarrow \infty} \int_{0}^{T} \int_{|x| \leq 2 T^{1 / \beta}} u^{p}(x, t) \tilde{\varphi}(x, t) d x d t=0 .
$$

Using the Lebesgue dominated convergence theorem, the continuity in time and space of $u$ and the fact that $\tilde{\varphi}(x, t) \rightarrow 1$ as $T \rightarrow \infty$, we infer that

$$
\int_{0}^{\infty} \int_{\mathbb{R}^{N}} u^{p}(x, t) d x d t=0 \quad \Longrightarrow \quad u \equiv 0
$$

Contradiction.

- The case $p=p^{*}(\delta=0)$. Using inequality (4.12) with $T \rightarrow \infty$ and taking into account the fact that $p=p^{*}$, we have on one hand

$$
u \in L^{p}\left((0, \infty), L^{p}\left(\mathbb{R}^{N}\right)\right) .
$$

On the other hand, repeating the same calculation as above by taking this time $\varphi_{1}(x):=$ $\Phi\left(|x| /\left(B^{-1 / \beta} T^{1 / \beta}\right)\right)$, where $1 \leq B<T$ is large enough such that when $T \rightarrow \infty$ we do not have $B \rightarrow \infty$ at the same time, we arrive at

$$
\int_{\Sigma_{T}} u^{p}(x, t) \tilde{\varphi}(x, t) \leq C B^{-N / \beta}+C B^{-N / \beta+\tilde{p}},
$$


thanks to the rescaling, $\tau=T^{-1} t, \xi=(T / B)^{-1 / \beta} x$, where

$$
\Sigma_{T}:=[0, T] \times\left\{x \in \mathbb{R}^{N} ;|x| \leq 2 B^{-1 / \beta} T^{1 / \beta}\right\} \quad \text { and } \quad \int_{\Sigma_{T}}=\int_{\Sigma_{T}} d x d t .
$$

Thus, using $p>N /(N-\beta)$ and taking the limits when $T \rightarrow \infty$ and then $B \rightarrow \infty$, we get

$$
\int_{0}^{\infty} \int_{\mathbb{R}^{N}} u^{p}(x, t) d x d t=0 \quad \Longrightarrow \quad u \equiv 0
$$

which is a contradiction.

Note that, in the case $\beta=2$ it is not necessary to take the condition $p>N /(N-\beta)$ with $N>\beta$. Indeed, from (4.9) with the new function $\varphi_{1}$, we may write

$$
\begin{aligned}
& \int_{\Sigma_{T}} u^{p}(x, t) \tilde{\varphi}(x, t) \\
& \leq C \int_{\Sigma_{T}} u(x, t) \tilde{\varphi}^{1 / p} \tilde{\varphi}^{-1 / p}\left(\varphi_{1}(x)\right)^{\ell}\left|D_{t \mid T}^{1+\alpha} \varphi_{2}(t)\right| \\
& +C \int_{\Delta_{B}} u(x, t) \tilde{\varphi}^{1 / p} \tilde{\varphi}^{-1 / p}\left(\varphi_{1}(x)\right)^{\ell-1}\left|\Delta_{x} \varphi_{1}(x) D_{t \mid T}^{\alpha} \varphi_{2}(t)\right|,
\end{aligned}
$$

where

$$
\Delta_{B}=[0, T] \times\left\{x \in \mathbb{R}^{N} \quad ; \quad B^{-1 / 2} T^{1 / 2} \leq|x| \leq 2 B^{-1 / 2} T^{1 / 2}\right\} \subset \Sigma_{T} \text { and } \int_{\Delta_{B}}=\int_{\Delta_{B}} d x d t .
$$

Moreover, using Young's inequality

$$
a b \leq \frac{1}{p} a^{p}+\frac{1}{\tilde{p}} b^{\tilde{p}}, \quad \text { where } p \tilde{p}=p+\tilde{p}, \quad a>0, b>0, \quad p>1, \tilde{p}>1,
$$

with

$$
\left\{\begin{array}{l}
a=u(x, t) \tilde{\varphi}^{1 / p} \\
b=\tilde{\varphi}^{-1 / p}\left(\varphi_{1}(x)\right)^{\ell}\left|D_{t \mid T}^{1+\alpha} \varphi_{2}(t)\right|
\end{array}\right.
$$

in the first integral of the right-hand side of (4.16) and the Hölder inequality

$$
\int_{\Delta_{B}} a b \leq\left(\int_{\Delta_{B}} a^{p}\right)^{1 / p}\left(\int_{\Delta_{B}} b^{\tilde{p}}\right)^{1 / \tilde{p}}, \quad \text { where } p \tilde{p}=p+\tilde{p}, a>0, b>0, \quad p>1, \tilde{p}>1
$$

with

$$
\left\{\begin{array}{l}
a=u(x, t) \tilde{\varphi}^{1 / p} \\
b=\tilde{\varphi}^{-1 / p}\left(\varphi_{1}(x)\right)^{\ell-1}\left|\Delta_{x} \varphi_{1}(x) D_{t \mid T}^{\alpha} \varphi_{2}(t)\right|
\end{array}\right.
$$

in the second integral of the right-hand side of (4.16), we obtain

$$
\begin{aligned}
& \left(1-\frac{1}{p}\right) \int_{\Sigma_{T}} u^{p}(x, t) \tilde{\varphi}(x, t) \\
& \leq C \int_{\Sigma_{T}}\left(\varphi_{1}(x)\right)^{\ell}\left(\varphi_{2}(t)\right)^{-\frac{1}{p-1}}\left|D_{t \mid T}^{1+\alpha} \varphi_{2}(t)\right|^{\tilde{p}} \\
& +C\left(\int_{\Delta_{B}} u^{p} \tilde{\varphi}\right)^{1 / p}\left(\int_{\Delta_{B}}\left(\varphi_{1}(x)\right)^{\ell-\tilde{p}}\left(\varphi_{2}(t)\right)^{-\frac{1}{p-1}}\left|\Delta_{x} \varphi_{1}(x) D_{t \mid T}^{\alpha} \varphi_{2}(t)\right|^{\tilde{p}}\right)^{1 / \tilde{p}} .
\end{aligned}
$$


Taking account of the scaled variables, $\tau=T^{-1} t, \xi=(T / B)^{-1 / 2} x$, and the fact that $\delta=0$, we get

$$
\int_{\Sigma_{T}} u^{p}(x, t) \tilde{\varphi}(x, t) \leq C B^{-N / 2}+C B^{-\frac{N}{2 \tilde{p}}+1}\left(\int_{\Delta_{B}} u^{p} \tilde{\varphi}\right)^{1 / p} .
$$

Now, as

$$
\lim _{T \rightarrow \infty}\left(\int_{\Delta_{B}} u^{p} \tilde{\varphi}\right)^{1 / p}=0 \quad(\text { from (4.14) }) \text {, }
$$

passing to the limit in (4.19) as $T \rightarrow \infty$, we get

$$
\int_{0}^{\infty} \int_{\mathbb{R}^{\mathbb{N}}} u^{p}(x, t) d x d t \leq C B^{-N / 2} .
$$

We conclude that $u \equiv 0$ by taking the limit when $B$ goes to infinity; contradiction.

- For the case $p<1 / \gamma$. We repeat the same argument as in the case $p<p^{*}$ by choosing this time the test function $\varphi(x, t)=D_{t \mid T}^{\alpha} \bar{\varphi}(x, t):=D_{t \mid T}^{\alpha}\left(\varphi_{3}^{\ell}(x) \varphi_{4}(t)\right)$, where $\varphi_{3}(x)=\Phi(|x| / R), \varphi_{4}(t)=(1-t / T)_{+}^{\eta}$ and $R \in(0, T)$ large enough such that when $T \rightarrow \infty$ we do not have $R \rightarrow \infty$ at the same time. The function $\Phi$ is the same as above. We then obtain

$$
\begin{aligned}
& \int_{\mathcal{C}_{T}} u^{p}(x, t) \bar{\varphi}(x, t)+C T^{-\alpha} \int_{\mathcal{C}}\left(\varphi_{3}(x)\right)^{\ell} u_{0}(x) \\
& \leq C \int_{\mathcal{C}_{T}} u(x, t) \bar{\varphi}^{1 / p} \bar{\varphi}^{-1 / p}\left(\varphi_{3}(x)\right)^{\ell}\left|D_{t \mid T}^{1+\alpha} \varphi_{4}(t)\right| \\
& +C \int_{\mathcal{C}_{T}} u(x, t) \bar{\varphi}^{1 / p} \bar{\varphi}^{-1 / p}\left(\varphi_{3}(x)\right)^{\ell-1}\left|\left(-\Delta_{x}\right)^{\beta / 2} \varphi_{3}(x) D_{t \mid T}^{\alpha} \varphi_{4}(t)\right|,
\end{aligned}
$$

where

$$
\mathcal{C}_{T}:=[0, T] \times \mathcal{C} \quad \text { for } \quad \mathcal{C}:=\left\{x \in \mathbb{R}^{N} ;|x| \leq 2 R\right\}, \quad \int_{\mathcal{C}}=\int_{\mathcal{C}} d x \text { and } \int_{\mathcal{C}_{T}}=\int_{\mathcal{C}_{T}} d x d t
$$

Now, by Young's inequality (4.10) with

$$
\left\{\begin{array}{l}
a=u(x, t) \bar{\varphi}^{1 / p} \\
b=\bar{\varphi}^{-1 / p}\left(\varphi_{3}(x)\right)^{\ell}\left|D_{t \mid T}^{1+\alpha} \varphi_{4}(t)\right|
\end{array}\right.
$$

in the first integral of the right-hand side of (4.20) and with

$$
\left\{\begin{array}{l}
a=u(x, t) \bar{\varphi}^{1 / p} \\
b=\bar{\varphi}^{-1 / p}\left(\varphi_{3}(x)\right)^{\ell-1}\left|\left(-\Delta_{x}\right)^{\beta / 2} \varphi_{3}(x) D_{t \mid T}^{\alpha} \varphi_{4}(t)\right|
\end{array}\right.
$$

in the second integral of the right-hand side of (4.20) and using the positivity of $u_{0}$, we get

$$
\begin{aligned}
\left(1-\frac{1}{p}\right) \int_{\mathcal{C}_{T}} u^{p}(x, t) \bar{\varphi}(x, t) & \leq C \int_{\mathcal{C}_{T}}\left(\varphi_{3}(x)\right)^{\ell}\left(\varphi_{4}(t)\right)^{-\frac{1}{p-1}}\left|D_{t \mid T}^{1+\alpha} \varphi_{4}(t)\right|^{\tilde{p}} \\
& +C \int_{\mathcal{C}_{T}}\left(\varphi_{3}(x)\right)^{\ell-\tilde{p}}\left(\varphi_{4}(t)\right)^{-\frac{1}{p-1}}\left|\left(-\Delta_{x}\right)^{\beta / 2} \varphi_{3} D_{t \mid T}^{\alpha} \varphi_{4}\right|^{\tilde{p}} .
\end{aligned}
$$


Then, the new variables $\xi=R^{-1} x, \tau=T^{-1} t$ and (2.14)-(2.15) allow us to obtain the estimate

$$
\int_{\mathcal{C}_{T}} u^{p}(x, t) \bar{\varphi}(x, t) d x d t \leq C T^{1-(1+\alpha) \tilde{p}} R^{N}+C T^{1-\alpha \tilde{p}} R^{N-\beta \tilde{p}} .
$$

Taking the limit as $T \rightarrow \infty$, we infer, as $p<1 / \gamma \Longleftrightarrow 1-\alpha \tilde{p}<0$, that

$$
\int_{0}^{\infty} \int_{\mathcal{C}} u(x, t)^{p}\left(\varphi_{3}(x)\right)^{\ell} d x d t=0 .
$$

Finally, by taking $R \rightarrow \infty$, we get a contradiction as $u(x, t)>0$ for all $x \in \mathbb{R}^{N}, t>0$.

REMARKs. (1) If we take $\beta=2$ and $v(x, t)=(\Gamma(1-\gamma))^{(1-\gamma) /(p-1)} u\left(\Gamma(1-\gamma)^{1 / 2} x, \Gamma(1-\right.$ $\gamma) t$ ), where $u$ is a solution of (1.1), we recover the result in [8] as a particular case.

(2) We can extend our analysis to the equation

$$
u_{t}=-(-\Delta)^{\beta / 2} u+\frac{1}{\Gamma(1-\gamma)} \int_{0}^{t} \frac{\psi(x, s)|u(s)|^{p-1} u(s)}{(t-s)^{\gamma}} d s, \quad x \in \mathbb{R}^{N},
$$

where $p>1, \beta \in(0,2], 0<\gamma<1$ and $\psi \in L_{\text {Loc }}^{1}\left(\mathbb{R}^{N} \times(0, \infty)\right), \psi(\cdot, t)>0$ for all $t>0$,

$$
\begin{cases}\psi\left(B^{-1 / \beta} T^{1 / \beta} \xi, T \tau\right) \geq C>0 & \text { if } p \leq p^{*} \\ \psi(R \xi, T \tau) \geq C>0 & \text { if } p<1 / \gamma\end{cases}
$$

for any $0<R, B<T, \tau \in[0,1]$ and $\xi \in[0,2]$.

(3) In Theorem 4.3. we use precisely the weak solution, but in this case we obtain a nonexistence of global weak solutions. Therefore, to obtain blow-up results, we use the mild solution and the alternative: either $T_{\max }=\infty$ or else $T_{\max }<\infty$ and $\|u\|_{L^{\infty}\left((0, t) \times \mathbb{R}^{N}\right)} \rightarrow \infty$ as $t \rightarrow T_{\max }$.

(4) We can take the nonlocal porous-medium spatio-fractional problem which is our first motivation to extend the results of $[8]$ :

$$
\begin{cases}u_{t}+(-\Delta)^{\beta / 2}|u|^{m-1} u=\frac{1}{\Gamma(1-\gamma)} \int_{0}^{t}(t-s)^{-\gamma}|u|^{p-1} u(s) d s & x \in \mathbb{R}^{N}, t>0 \\ u(x, 0)=u_{0}(x) & x \in \mathbb{R}^{N},\end{cases}
$$

where $\beta \in(0,2], 0<\gamma<1,1 \leq m<p, u_{0} \geq 0$ and $u_{0} \not \equiv 0$.

The threshold on $p$ will be

$$
p \leq 1+\frac{(2-\gamma)(N(m-1)+\beta)}{(N-\beta+\beta \gamma)_{+}} \quad \text { or } \quad p<\frac{m}{\gamma} .
$$

5. Blow-up rate in the case $\beta=2$. In this section, we present the blow-up rate for the blowing-up solutions to the parabolic problem (1.1).

We take the solution of (1.1) with an initial condition satisfying

$$
u_{0} \in C_{0}\left(\mathbb{R}^{N}\right) \cap L^{2}\left(\mathbb{R}^{N}\right), \quad u(\cdot, 0)=u_{0} \geq 0, \quad u_{0} \not \equiv 0 .
$$

The following lemma will be used in the proof of Theorem 5.2 below. 
LEMma 5.1. Let $\varphi$ be a nonnegative classical solution of

$$
\varphi_{t}=\Delta \varphi+J_{-\infty \mid t}^{1-\gamma}\left(\varphi^{p}\right) \quad \text { in } \mathbb{R}^{N} \times \mathbb{R},
$$

where $\gamma \in(0,1), p>1$ and

$$
J_{-\infty \mid t}^{1-\gamma}\left(\varphi^{p}\right)(t):=\frac{1}{\Gamma(1-\gamma)} \int_{-\infty}^{t}(t-s)^{-\gamma} \varphi^{p}(s) d s .
$$

Then $\varphi \equiv 0$ whenever

$$
p \leq p^{*} \quad \text { or } \quad p<\frac{1}{\gamma} .
$$

Proof. We repeat the same computations as in Theorem 4.3 with $\left(1-t^{2} / T^{2}\right)_{+}^{\eta}$ instead of $(1-t / T)_{+}^{\eta}$ for $\eta \gg 1$, use (2.17)-(2.19) and take account of the inequality

$$
J_{-\infty \mid t}^{1-\gamma}\left(\varphi^{p}\right) \geq J_{-T \mid t}^{1-\gamma}\left(\varphi^{p}\right) .
$$

Moreover, we take $\varphi_{1}^{\ell / p} \varphi_{1}^{-\ell / p}$ instead of $\tilde{\varphi}^{1 / p} \tilde{\varphi}^{-1 / p}$ (resp. $\left.\bar{\varphi}^{1 / p} \bar{\varphi}^{-1 / p}\right)$ in (4.9), (4.16) (resp. in (4.20) for $\ell \gg 1$ to use the Young and Hölder inequalities.

Note that here, we rather use the $\varepsilon$-Young inequality

$$
a b \leq \frac{\varepsilon}{2} a^{p}+C(\varepsilon) b^{\widetilde{p}}
$$

for $0<\varepsilon<1$.

Theorem 5.2. Let $u_{0}$ satisfy (5.1). For $p \leq p^{*}$ or $p<(1 / \gamma)$, let $\alpha_{1}:=(2-\gamma) /(p-1)$, and let $u$ be the blowing-up mild solution of (1.1) in a finite time $T_{\max }:=T^{*}$. Then there exist two constants $c, C>0$ such that

$$
c\left(T^{*}-t\right)^{-\alpha_{1}} \leq \sup _{\mathbb{R}^{N}} u(\cdot, t) \leq C\left(T^{*}-t\right)^{-\alpha_{1}}, \quad t \in\left(0, T^{*}\right) .
$$

Proof. The proof is in two parts:

- The upper blow-up rate estimate. Let

$$
M(t):=\sup _{\mathbb{R}^{N} \times(0, t]} u, \quad t \in\left(0, T^{*}\right) .
$$

Clearly, $M$ is positive, continuous, and nondecreasing in $\left(0, T^{*}\right)$. As $\lim _{t \rightarrow T^{*}} M(t)=\infty$, then for all $t_{0} \in\left(0, T^{*}\right)$, we can define

$$
t_{0}^{+}:=t^{+}\left(t_{0}\right):=\max \left\{t \in\left(t_{0}, T^{*}\right): M(t)=2 M\left(t_{0}\right)\right\} .
$$

Choose $A \geq 1$, and let

$$
\lambda\left(t_{0}\right):=\left(\frac{1}{2 A} M\left(t_{0}\right)\right)^{-1 /\left(2 \alpha_{1}\right)} .
$$

We claim that

$$
\lambda^{-2}\left(t_{0}\right)\left(t_{0}^{+}-t_{0}\right) \leq D, \quad t_{0} \in\left(\frac{T^{*}}{2}, T^{*}\right),
$$

where $D>0$ is a positive constant which does not depend on $t_{0}$.

We proceed by contradiction. If (5.6) were false, then there would exist a sequence $t_{n} \rightarrow T^{*}$ such that

$$
\lambda_{n}^{-2}\left(t_{n}^{+}-t_{n}\right) \longrightarrow \infty,
$$


where $\lambda_{n}=\lambda\left(t_{n}\right)$ and $t_{n}^{+}=t^{+}\left(t_{n}\right)$. For each $t_{n}$ choose

$$
\left(\hat{x}_{n}, \hat{t}_{n}\right) \in \mathbb{R}^{N} \times\left(0, t_{n}\right] \text { such that } u\left(\hat{x}_{n}, \hat{t}_{n}\right) \geq \frac{1}{2} M\left(t_{n}\right) .
$$

Obviously, $M\left(t_{n}\right) \rightarrow \infty$; hence, $\hat{t}_{n} \rightarrow T^{*}$. Next, rescale the function $u$ as

$$
\varphi^{\lambda_{n}}(y, s):=\lambda_{n}^{2 \alpha_{1}} u\left(\lambda_{n} y+\hat{x}_{n}, \lambda_{n}^{2} s+\hat{t}_{n}\right), \quad(y, s) \in \mathbb{R}^{N} \times I_{n}\left(T^{*}\right),
$$

where $I_{n}(t):=\left(-\lambda_{n}^{-2} \hat{t}_{n}, \lambda_{n}^{-2}\left(t-\hat{t}_{n}\right)\right)$ for all $t>0$. Then $\varphi^{\lambda_{n}}$ is a mild solution of

$$
\varphi_{s}=\Delta \varphi+J_{-\lambda_{n}^{-2} \hat{t}_{n} \mid s}^{\alpha}\left(\varphi^{p}\right) \quad \text { in } \mathbb{R}^{N} \times I_{n}\left(T^{*}\right),
$$

i.e., for $G(t):=G(x, t):=(4 \pi t)^{-N / 2} e^{-|x|^{2} / 4 t}$ and $*$ being the space convolution, we have

$$
\varphi^{\lambda_{n}}(s)=G\left(s+\lambda_{n}^{-2} \hat{t}_{n}\right) * \varphi^{\lambda_{n}}\left(-\lambda_{n}^{-2} \hat{t}_{n}\right)+\int_{-\lambda_{n}^{-2} \hat{t}_{n}}^{s} G(s-\sigma) * J_{-\lambda_{n}^{-2} \hat{t}_{n} \mid \sigma}^{\alpha}\left(\left(\varphi^{\lambda_{n}}\right)^{p}\right) d \sigma
$$

in $\mathbb{R}^{N} \times I_{n}\left(T^{*}\right)$. Whereupon, as $\varphi^{\lambda_{n}}(0,0) \geq A$,

$$
0 \leq \varphi^{\lambda_{n}} \leq \lambda_{n}^{2 \alpha_{1}} M\left(t_{n}^{+}\right)=\lambda_{n}^{2 \alpha_{1}} 2 M\left(t_{n}\right)=4 A \quad \text { in } \mathbb{R}^{N} \times I_{n}\left(t_{n}^{+}\right),
$$

thanks to (5.5) and the definition of $t_{n}^{+}$.

Moreover, as

$$
\varphi^{\lambda_{n}} \in C\left(\left[-\lambda_{n}^{-2} \hat{t}_{n}, T\right], C_{0}\left(\mathbb{R}^{N}\right) \cap L^{2}\left(\mathbb{R}^{N}\right)\right) \quad \text { for all } T \in I_{n}\left(T^{*}\right),
$$

so, as in Lemma 4.2. $\varphi^{\lambda_{n}}$ is a weak solution of (5.9).

On the other hand, if we write $\varphi^{\lambda_{n}}$ as $\varphi^{\lambda_{n}}(s)=v(s)+w(s)$ for all $s \in I_{n}\left(T^{*}\right)$, where $v(s):=G\left(s+\lambda_{n}^{-2} \hat{t_{n}}\right) * \varphi^{\lambda_{n}}\left(-\lambda_{n}^{-2} \hat{t}_{n}\right) \quad$ and $\quad w(s):=\int_{-\lambda_{n}^{-2} \hat{t}_{n}}^{s} G(s-\sigma) * J_{-\lambda_{n}^{-2} \hat{t}_{n} \mid \sigma}^{\alpha}\left(\left(\varphi^{\lambda_{n}}\right)^{p}\right) d \sigma$ we have (see [6, Chapter 3]) for $T \in I_{n}\left(T^{*}\right)$

$$
v \in C\left(\left(-\lambda_{n}^{-2} \hat{t}_{n}, T\right) ; H^{2}\left(\mathbb{R}^{N}\right)\right) \cap C^{1}\left(\left(-\lambda_{n}^{-2} \hat{t}_{n}, T\right) ; L^{2}\left(\mathbb{R}^{N}\right)\right) \subset L^{2}\left(\left(-\lambda_{n}^{-2} \hat{t}_{n}, T\right) ; H^{1}\left(\mathbb{R}^{N}\right)\right)
$$

and, using the fact that $f(s):=J_{-\lambda_{n}^{-2} \hat{t}_{n} \mid s}^{\alpha}\left(\left(\varphi^{\lambda_{n}}\right)^{p}\right) \in L^{2}\left(\left(-\lambda_{n}^{-2} \hat{t}_{n}, T\right) ; L^{2}\left(\mathbb{R}^{N}\right)\right)$ and the maximal regularity theory, we have

$$
w \in W^{1,2}\left(\left(-\lambda_{n}^{-2} \hat{t}_{n}, T\right) ; L^{2}\left(\mathbb{R}^{N}\right)\right) \cap L^{2}\left(\left(-\lambda_{n}^{-2} \hat{t}_{n}, T\right) ; H^{2}\left(\mathbb{R}^{N}\right)\right) \subset L^{2}\left(\left(-\lambda_{n}^{-2} \hat{t}_{n}, T\right) ; H^{1}\left(\mathbb{R}^{N}\right)\right) .
$$

It follows that

$$
\varphi^{\lambda_{n}} \in C\left(\left[-\lambda_{n}^{-2} \hat{t}_{n}, T\right], L^{2}\left(\mathbb{R}^{N}\right)\right) \cap L^{2}\left(\left(-\lambda_{n}^{-2} \hat{t}_{n}, T\right), W^{1,2}\left(\mathbb{R}^{N}\right)\right) ;
$$

so from the parabolic interior regularity theory (cf. [22, Theorem 10.1, p. 204]) there is $\mu \in(0,1)$ such that the sequence $\varphi^{\lambda_{n}}$ is bounded in the $C_{\text {loc }}^{\mu, \mu / 2}\left(\mathbb{R}^{N} \times \mathbb{R}\right)$-norm by a constant independent of $n$, where $C_{\mathrm{loc}}^{\mu, \mu / 2}\left(\mathbb{R}^{N} \times \mathbb{R}\right)$ is the local Hölder space defined in 22. Similar uniform estimates for $J_{-\lambda_{n}^{-2} \hat{t}_{n} \mid s}^{\alpha}\left(\varphi^{p}\right)$ follow if $\mu$ is sufficiently small. The parabolic interior Schauder's estimates (see [21, Th. 8.11.1 p. 130]), using the existence theorems in the Hölder space, imply now that the $C_{\mathrm{loc}}^{2+\mu, 1+\mu / 2}\left(\mathbb{R}^{N} \times \mathbb{R}\right)$-norm of $\varphi^{\lambda_{n}}$ is uniformly bounded. Hence, we obtain a subsequence converging in $C_{\text {loc }}^{2+\mu, 1+\mu / 2}\left(\mathbb{R}^{N} \times \mathbb{R}\right)$ to a solution $\varphi$ of

$$
\varphi_{s}=\Delta \varphi+J_{-\infty \mid s}^{\alpha}\left(\varphi^{p}\right) \quad \text { in } \mathbb{R}^{N} \times(-\infty,+\infty),
$$


such that $\varphi(0,0) \geq A$ and $0 \leq \varphi \leq 4 A$ in $\mathbb{R}^{N} \times \mathbb{R}$. Whereupon, using Lemma 5.1] we infer that $\varphi \equiv 0$ in $\mathbb{R}^{N} \times(-\infty,+\infty)$. Contradiction with the fact that $\varphi(0,0) \geq A>1$. This proves (5.6).

Next we use an idea from $\mathrm{Hu}$ [15. From (5.5) and (5.6) it follows that

$$
\left(t_{0}^{+}-t_{0}\right) \leq D(2 A)^{1 / \alpha_{1}} M\left(t_{0}\right)^{-1 / \alpha_{1}} \quad \text { for any } t_{0} \in\left(\frac{T^{*}}{2}, T^{*}\right) \text {. }
$$

Fix $t_{0} \in\left(T^{*} / 2, T^{*}\right)$ and denote $t_{1}=t_{0}^{+}, t_{2}=t_{1}^{+}, t_{3}=t_{2}^{+}, \ldots$ Then

$$
\begin{aligned}
t_{j+1}-t_{j} & \leq D(2 A)^{1 / \alpha_{1}} M\left(t_{j}\right)^{-1 / \alpha_{1}}, \\
M\left(t_{j+1}\right) & =2 M\left(t_{j}\right),
\end{aligned}
$$

$j=0,1,2, \ldots$ Consequently,

$$
\begin{aligned}
T^{*}-t_{0} & =\sum_{j=0}^{\infty}\left(t_{j+1}-t_{j}\right) \leq D(2 A)^{1 / \alpha_{1}} \sum_{j=0}^{\infty} M\left(t_{j}\right)^{-1 / \alpha_{1}} \\
& =D(2 A)^{1 / \alpha_{1}} M\left(t_{0}\right)^{-1 / \alpha_{1}} \sum_{j=0}^{\infty} 2^{-j / \alpha_{1}} .
\end{aligned}
$$

Finally, we conclude that

$$
u\left(x, t_{0}\right) \leq M\left(t_{0}\right) \leq C\left(T^{*}-t_{0}\right)^{-\alpha_{1}}, \quad \forall t_{0} \in\left(0, T^{*}\right)
$$

where

SO

$$
C=2 A\left(D \sum_{j=0}^{\infty} 2^{-j / \alpha_{1}}\right)^{\alpha_{1}}
$$

$$
\sup _{\mathbb{R}^{N}} u(\cdot, t) \leq C\left(T^{*}-t\right)^{-\alpha_{1}}, \quad \forall t \in\left(0, T^{*}\right) .
$$

- The lower blow-up rate estimate. If we repeat the proof of the local existence of Theorem 3.2, by taking $\|u\|_{1} \leq \theta$ instead of $\|u\|_{1} \leq 2\left\|u_{0}\right\|_{\infty}$ in the space $E_{T}$ for all positive constant $\theta>0$ and all $0<t<T$, then the condition (3.2) of $T$ will be

$$
\left\|u_{0}\right\|_{\infty}+C T^{2-\gamma} \theta^{p} \leq \theta
$$

and then, as before, we infer that $\|u(t)\|_{\infty} \leq \theta$ for (almost) all $0<t<T$. Consequently, if $\left\|u_{0}\right\|_{\infty}+C t^{2-\gamma} \theta^{p} \leq \theta$, then $\|u(t)\|_{\infty} \leq \theta$. Applying this to any point in the trajectory, we see that if $0 \leq s<t$ and

$$
(t-s)^{2-\gamma} \leq \frac{\theta-\|u(s)\|_{\infty}}{C \theta^{p}},
$$

then $\|u(t)\|_{\infty} \leq \theta$, for all $0<t<T$.

Moreover, if $0 \leq s<T^{*}$ and $\|u(s)\|_{\infty}<\theta$, then

$$
\left(T^{*}-s\right)^{2-\gamma}>\frac{\theta-\|u(s)\|_{\infty}}{C \theta^{p}} .
$$

Indeed, arguing by contradiction and assuming that for some $\theta>\|u(s)\|_{\infty}$ and all $t \in$ $\left(s, T^{*}\right)$, we have

$$
(t-s)^{2-\gamma} \leq \frac{\theta-\|u(s)\|_{\infty}}{C \theta^{p}} .
$$


Then, using (5.12), we infer that $\|u(t)\|_{\infty} \leq \theta$ for all $t \in\left(s, T^{*}\right)$. This contradicts the fact that $\|u(t)\|_{\infty} \rightarrow \infty$ as $t \rightarrow T^{*}$.

Next, for example, by setting $\theta=2\|u(s)\|_{\infty}$ in (5.13), we see that for $0<s<T^{*}$ we have

$$
\left(T^{*}-s\right)^{2-\gamma}>C^{\prime}\|u(s)\|_{\infty}^{1-p},
$$

and by the positivity and the continuity of $u$ we get

$$
c\left(T^{*}-s\right)^{-\alpha_{1}}<\sup _{x \in \mathbb{R}^{N}} u(x, s), \quad \forall s \in\left(0, T^{*}\right) .
$$

6. Global existence. In this section, we prove the existence of global solutions of (1.1) with initial data small enough. We give a similar proof as that in 8 just for the sake of completeness. In the following, we use the notation $p_{s c}:=N(p-1) / \beta(2-\gamma)$. As $p^{*}>1+\beta(2-\gamma) / N$, we note that $p>p^{*} \Rightarrow p_{s c}>1$.

Theorem 6.1. Let $u_{0} \in C_{0}\left(\mathbb{R}^{N}\right) \cap L^{p_{s c}}\left(\mathbb{R}^{N}\right)$ and $0<\beta \leq 2$. If

$$
p>\max \left\{\frac{1}{\gamma} ; p^{*}\right\}
$$

and $\left\|u_{0}\right\|_{L^{p_{s c}}}$ is sufficiently small, then the mild solution $u$ of 1.1 exists globally.

Note that we can take $\left|u_{0}(x)\right| \leq C|x|^{-\beta(2-\gamma) /(p-1)}$ instead of $u_{0} \in L^{p_{s c}}\left(\mathbb{R}^{N}\right)$.

Proof. As $p>(1 / \gamma)$, then we have the possibility to take a positive constant $q>0$ so that

$$
\frac{2-\gamma}{p-1}-\frac{1}{p}<\frac{N}{\beta q}<\frac{1}{p-1}, \quad q \geq p .
$$

It follows, using (6.1), that

$$
q>\frac{N(p-1)}{\beta}>p_{s c}>1
$$

Let

$$
b:=\frac{N}{\beta p_{s c}}-\frac{N}{\beta q}=\frac{2-\gamma}{p-1}-\frac{N}{\beta q} .
$$

Then, using (6.2)-(6.4), we conclude that

$$
b>\frac{1-\gamma}{p-1}>0, \quad p b<1, \quad \frac{N(p-1)}{\beta q}+(p-1) b+\gamma=2 .
$$

As $u_{0} \in L^{p_{s c}}$, using (2.4) and (6.4), we get, for all $t>0$,

$$
\sup _{t>0} t^{b}\left\|e^{-t(-\Delta)^{\beta / 2}} u_{0}\right\|_{L^{q}} \leq C\left\|u_{0}\right\|_{L^{p_{s c}}}=\eta<\infty .
$$

Set

$$
\Xi:=\left\{u \in L^{\infty}\left((0, \infty), L^{q}\left(\mathbb{R}^{N}\right)\right) ; \sup _{t>0} t^{b}\|u(t)\|_{L^{q}} \leq \delta\right\},
$$

where $\delta>0$ is to be chosen sufficiently small. If we define

$$
d_{\Xi}(u, v):=\sup _{t>0} t^{b}\|u(t)-v(t)\|_{L^{q}}, \quad \forall u, v \in \Xi,
$$


then $(\Xi, d)$ is a complete metric space. Given $u \in \Xi$, let us set

$$
\Phi(u)(t):=e^{-t(-\Delta)^{\beta / 2}} u_{0}+\frac{1}{\Gamma(1-\gamma)} \int_{0}^{t} e^{-(t-s)(-\Delta)^{\beta / 2}} \int_{0}^{s}(s-\sigma)^{-\gamma}|u|^{p-1} u(\sigma) d \sigma d s,
$$

for all $t \geq 0$. We have by (2.4), (6.6), and (6.7)

$$
\begin{aligned}
t^{b}\|\Phi(u)(t)\|_{L^{q}} & \leq \eta+C t^{b} \int_{0}^{t}(t-s)^{-\frac{N}{\beta}\left(\frac{p}{q}-\frac{1}{q}\right)} \int_{0}^{s}(s-\sigma)^{-\gamma}\left\|u^{p}(\sigma)\right\|_{L^{\frac{q}{p}}} d \sigma d s \\
& \leq \eta+C \delta^{p} t^{b} \int_{0}^{t} \int_{0}^{s}(t-s)^{-\frac{N(p-1)}{\beta q}}(s-\sigma)^{-\gamma} \sigma^{-b p} d \sigma d s .
\end{aligned}
$$

Next, using (6.2) and $p b<1$, we get

$$
\begin{aligned}
\int_{0}^{t} \int_{0}^{s} \frac{(t-s)^{-\frac{N}{\beta q}(p-1)}}{(s-\sigma)^{\gamma}} \sigma^{-b p} d \sigma d s & =\left(\int_{0}^{1}(1-\sigma)^{-\gamma} \sigma^{-b p} d \sigma\right) \int_{0}^{t} \frac{(t-s)^{-\frac{N(p-1)}{\beta q}}}{s^{b p+\gamma-1}} d s \\
& =C t^{-\frac{N(p-1)}{\beta q}-b p-\gamma+2}=C t^{-b}
\end{aligned}
$$

for all $t \geq 0$. So, we deduce from (6.10), (6.11) that

$$
t^{b}\|\Phi(u)(t)\|_{L^{q}} \leq \eta+C \delta^{p} .
$$

Therefore, if $\eta$ and $\delta$ are chosen small enough so that $\eta+C \delta^{p} \leq \delta$, we see that $\Phi: \Xi \rightarrow \Xi$. Similar calculations show that (assuming $\eta$ and $\delta$ small enough) $\Phi$ is a strict contraction, so it has a unique fixed point $u \in \Xi$ which is a solution of (1.1). Now, we show that $u \in C\left([0, \infty), C_{0}\left(\mathbb{R}^{N}\right)\right)$.

First, we show that $u \in C\left([0, T], C_{0}\left(\mathbb{R}^{N}\right)\right)$ if $T>0$ is sufficiently small. Indeed, note that the above argument shows uniqueness in $\Xi_{T}$, where, for any $T>0$,

$$
\Xi_{T}:=\left\{u \in L^{\infty}\left((0, T), L^{q}\left(\mathbb{R}^{N}\right)\right) ; \sup _{0<t<T} t^{b}\|u(t)\|_{L^{q}} \leq \delta\right\} .
$$

Let $\tilde{u}$ be the local solution of (1.1) constructed in Theorem 3.2. Since $u_{0} \in C_{0}\left(\mathbb{R}^{N}\right) \cap$ $L^{p_{s c}}\left(\mathbb{R}^{N}\right)$, then, using the fact that $u_{0} \in L^{q}\left(\mathbb{R}^{N}\right)$ and (6.3), we have $\tilde{u} \in C\left(\left[0, T_{\max }\right)\right.$, $\left.L^{q}\left(\mathbb{R}^{N}\right)\right)$ by Theorem 3.2. It follows that $\sup _{0<t<T} t^{b}\|\tilde{u}(t)\|_{L^{q}} \leq \delta$ if $T>0$ is sufficiently small. Therefore, by uniqueness, $u=\tilde{u}$ on $[0, T]$, so that $u \in C\left([0, T], C_{0}\left(\mathbb{R}^{N}\right)\right)$.

Next, we show that $u \in C\left([T, \infty), C_{0}\left(\mathbb{R}^{N}\right)\right)$ by a bootstrap argument. Indeed, for $t>T$, we write

$$
\begin{aligned}
u(t)-e^{-t(-\Delta)^{\beta / 2}} u_{0} & =\int_{0}^{t} e^{-(t-s)(-\Delta)^{\beta / 2}} \int_{0}^{T}(s-\sigma)^{-\gamma}|u|^{p-1} u(\sigma) d \sigma d s \\
& +\int_{0}^{t} e^{-(t-s)(-\Delta)^{\beta / 2}} \int_{T}^{s}(s-\sigma)^{-\gamma}|u|^{p-1} u(\sigma) d \sigma d s \\
& \equiv I_{1}(t)+I_{2}(t) .
\end{aligned}
$$

Since $u \in C\left([0, T], C_{0}\left(\mathbb{R}^{N}\right)\right)$, it follows that $I_{1} \in C\left([T, \infty), C_{0}\left(\mathbb{R}^{N}\right)\right)$. Also, by the calculations used to construct the fixed point, using the fact that $t^{-b} \leq T^{-b}<\infty$ and $p q>q$, $I_{1} \in C\left([T, \infty), L^{q}\left(\mathbb{R}^{N}\right)\right)$. Next, note that $N(p / q-1 / q) / \beta<1$ by (6.3); therefore, there exists $r \in(q, \infty]$ such that

$$
\frac{N}{\beta}\left(\frac{p}{q}-\frac{1}{r}\right)<1
$$


Let $T<s<t$ (the case of $s \leq T \leq t$ is obvious). Since $u \in L^{\infty}\left((0, \infty), L^{q}\left(\mathbb{R}^{N}\right)\right)$, we have $|u|^{p-1} u \in L^{\infty}\left((T, s), L^{q / p}\left(\mathbb{R}^{N}\right)\right)$, and it easily follows, using (2.4) and (6.13), that $I_{2} \in C\left([T, \infty), L^{r}\left(\mathbb{R}^{N}\right)\right)$. As $e^{-\cdot(-\Delta)^{\beta / 2}} u_{0}$ and $I_{1}$ both belong to $C\left([T, \infty), C_{0}\left(\mathbb{R}^{N}\right)\right) \cap$ $C\left([T, \infty), L^{q}\left(\mathbb{R}^{N}\right)\right)$, we see that $u \in C\left([T, \infty), L^{r}\left(\mathbb{R}^{N}\right)\right)$. Iterating this procedure a finite number of times, we deduce that $u \in C\left([T, \infty), C_{0}\left(\mathbb{R}^{N}\right)\right)$. This completes the proof.

7. Necessary conditions for local or global existence. In this section, we establish necessary conditions for the existence of local or global weak solutions to the problem (1.1). These conditions depend on the behavior of the initial data for large $x$.

Theorem 7.1 (Necessary conditions for global existence). Let $u_{0} \in L_{\mathrm{Loc}}^{\infty}\left(\mathbb{R}^{N}\right), u_{0} \geq 0$, $0<\beta \leq 2$ and $p>1$. If $u$ is a global weak solution to problem (1.1), then there is a positive constant $C>0$ such that

$$
\liminf _{|x| \rightarrow \infty}\left(u_{0}(x)|x|^{\frac{\beta(2-\gamma)}{p-1}}\right) \leq C .
$$

Proof. Let $u$ be a global weak solution to (1.1), then $u \in L^{p}\left(\left(0, R^{\beta}\right), L^{\infty}\left(B_{2 R}\right)\right)$ for all $R>0$, where $B_{2 R}$ stands for the closed ball of center 0 and radius $2 R$. So, we repeat the same calculation as in the proof of Theorem 3.2 (here in bounded domain) by taking $\varphi(x, t):=D_{t \mid T}^{\alpha} \tilde{\varphi}(x, t):=D_{t \mid T}^{\alpha}\left(\varphi_{1}(x / R) \varphi_{2}(t)\right)$ instead of the one chosen in Theorem 3.2 , where $0 \leq \varphi_{1} \in D\left(\Delta_{D}^{\beta / 2}\right)$ is the first eigenfunction of the fractional Laplacian operator $\Delta_{D}^{\beta / 2}$ in $B_{2}$, with the homogeneous Dirichlet boundary condition (2.6), associated to the first eigenvalue $\lambda:=\lambda_{1}^{\beta / 2}$, and $\varphi_{2}(t):=\left(1-t / R^{\beta}\right)_{+}^{\ell}$ for $\ell \gg 1$ large enough.

Then, as for the estimate (4.11), we obtain, with $\Sigma:=\left[0, R^{\beta}\right] \times B_{2 R}$,

$$
\begin{aligned}
& \int_{\Sigma} u^{p} \tilde{\varphi} d x d t+C R^{-\alpha \beta} \int_{|x| \leq 2 R} u_{0}(x) \varphi_{1}(x / R) d x \\
& \leq C \int_{\Sigma} \varphi_{1}(x / R)\left(\varphi_{2}(t)\right)^{-\frac{1}{p-1}}\left|D_{t \mid R^{\beta}}^{1+\alpha} \varphi_{2}(t)\right|^{\tilde{p}} d x d t \\
& +C \int_{\Sigma}\left(\varphi_{1}(x / R)\right)^{-\frac{1}{p-1}}\left(\varphi_{2}(t)\right)^{-\frac{1}{p-1}}\left|\Delta_{D}^{\beta / 2} \varphi_{1}(x / R) D_{t \mid R^{\beta}}^{\alpha} \varphi_{2}(t)\right|^{\tilde{p}} d x d t,
\end{aligned}
$$

where $\alpha:=1-\gamma$ and $\tilde{p}:=p /(p-1)$. If we take the scaled variables $\tau=t / R^{\beta}, \xi=x / R$ and use the fact that $\Delta_{D}^{\beta / 2} \varphi_{1}(x / R)=R^{-\beta} \lambda \varphi_{1}(x / R)$ in the right-hand side of (7.2), taking into account the positivity of $u$, we infer that

$$
\begin{aligned}
& C R^{-\alpha \beta} \int_{|\xi| \leq 2} u_{0}(R \xi) \varphi_{1}(\xi) d \xi \\
& \leq C(R) \int_{|\xi| \leq 2} \varphi_{1}(\xi) d \xi \\
& =C(R) \int_{|\xi| \leq 2}|R \xi|^{\beta(1+\alpha)(\tilde{p}-1)}|R \xi|^{\beta(1+\alpha)(1-\tilde{p})} \varphi_{1}(\xi) d \xi \\
& \leq C(R)(2 R)^{\beta(1+\alpha)(\tilde{p}-1)} \int_{|\xi| \leq 2}|R \xi|^{\beta(1+\alpha)(1-\tilde{p})} \varphi_{1}(\xi) d \xi,
\end{aligned}
$$


where $\bar{C}(R)=R^{\beta-(1+\alpha) \beta \tilde{p}}(C+C \lambda)$, and so

$$
\int_{|\xi| \leq 2} u_{0}(R \xi) \varphi_{1}(\xi) d \xi \leq C \int_{|\xi| \leq 2}|R \xi|^{\beta(1+\alpha)(1-\tilde{p})} \varphi_{1}(\xi) d \xi
$$

Using the estimate

$$
\begin{aligned}
\inf _{|\xi|>1}\left(u_{0}(R \xi)|R \xi|^{\beta(1+\alpha)(\tilde{p}-1)}\right) \int_{|\xi| \leq 2}|R \xi|^{\beta(1+\alpha)(1-\tilde{p})} \varphi_{1}(\xi) d \xi & \leq \int_{1<|\xi| \leq 2} u_{0}(R \xi) \varphi_{1}(\xi) d \xi \\
& \leq \int_{|\xi| \leq 2} u_{0}(R \xi) \varphi_{1}(\xi) d \xi
\end{aligned}
$$

in the left-hand side of (7.3) , we conclude, after dividing by $\int_{|\xi| \leq 2}|R \xi|^{\beta(1+\alpha)(1-\tilde{p})} \varphi_{1}(\xi) d \xi$ that

$$
\inf _{|\xi|>1}\left(u_{0}(R \xi)|R \xi|^{\beta(1+\alpha)(\tilde{p}-1)}\right) \leq C .
$$

Passing to the limit in (17.4), as $R \rightarrow \infty$, we obtain

$$
\liminf _{|x| \rightarrow \infty}\left(u_{0}(x)|x|^{\beta(1+\alpha)(\tilde{p}-1)}\right) \leq C .
$$

Corollary 1 (Sufficient conditions for the nonexistence of global solutions). Let $u_{0} \in$ $L_{\text {Loc }}^{\infty}\left(\mathbb{R}^{N}\right), u_{0} \geq 0,0<\beta \leq 2$, and $p>1$. If

$$
\liminf _{|x| \rightarrow \infty}\left(u_{0}(x)|x|^{\frac{\beta(2-\gamma)}{p-1}}\right)=+\infty
$$

then problem (1.1) cannot admit a global weak solution.

Next, we give a necessary condition for local existence where we obtain a similar estimate of $T$ found in the proof of Theorem [3.2, as $|x|$ goes to infinity.

TheOREM 7.2 (Necessary conditions for local existence). Let $u_{0} \in L_{\text {Loc }}^{\infty}\left(\mathbb{R}^{N}\right), u_{0} \geq 0$, $\beta \in(0,2]$, and $p>1$. If $u$ is a local weak solution to problem (1.1) on $[0, T]$ where $0<T<+\infty$, then we have

$$
\liminf _{|x| \rightarrow \infty} u_{0}(x) \leq C T^{-\frac{2-\gamma}{p-1}}
$$

for some positive constant $C>0$.

Note that, if $A:=\liminf _{|x| \rightarrow \infty} u_{0}(x)$, then we obtain a similar estimate as that found in (3.4),

$$
\frac{T^{2-\gamma} A^{p-1}}{C^{p-1}} \leq 1
$$

Proof. We take here, for $R>0$ sufficiently large, $\varphi(x, t):=D_{t \mid T}^{\alpha} \tilde{\varphi}(x, t):=D_{t \mid T}^{\alpha}$ $\left(\varphi_{1}(x / R) \varphi_{2}(t)\right)$ where $\varphi_{2}(t):=(1-t / T)_{+}^{\ell}$ instead of the one chosen in Theorem 7.1 . 
Then, as (7.2), we obtain

$$
\begin{aligned}
& \int_{\Sigma_{1}} u^{p} \tilde{\varphi} d x d t+C T^{-\alpha} \int_{|x| \leq 2 R} u_{0}(x) \varphi_{1}(x / R) d x \\
& \leq C \int_{\Sigma_{1}} \varphi_{1}(x / R)\left(\varphi_{2}(t)\right)^{-\frac{1}{p-1}}\left|D_{t \mid T}^{1+\alpha} \varphi_{2}(t)\right|^{\tilde{p}} d x d t \\
& +C \int_{\Sigma_{1}}\left(\varphi_{1}(x / R)\right)^{-\frac{2}{p-1}}\left(\varphi_{2}(t)\right)^{-\frac{1}{p-1}}\left|\Delta_{D}^{\beta / 2} \varphi_{1}(x / R) D_{t \mid T}^{\alpha} \varphi_{2}(t)\right|^{\tilde{p}} d x d t,
\end{aligned}
$$

where $\Sigma_{1}:=[0, T] \times\left\{x \in \mathbb{R}^{N} ;|x| \leq 2 R\right\}, \alpha:=1-\gamma$ and $\tilde{p}:=p /(p-1)$. Now, in the right-hand side of (7.6), we take the scaled variables $\tau=T^{-1} t, \xi=R^{-1} x$ and use the fact that $\Delta_{D}^{\beta / 2} \varphi_{1}(x / R)=R^{-\beta} \lambda \varphi_{1}(x / R)$, while in the left-hand side we use the positivity of $u$. Then we get

$$
C T^{-\alpha} \int_{|\xi| \leq 2} u_{0}(R \xi) \varphi_{1}(\xi) d \xi \leq\left(C T^{1-(1+\alpha) \tilde{p}}+C \lambda T^{1-\alpha \tilde{p}} R^{-\beta \tilde{p}}\right) \int_{|\xi| \leq 2} \varphi_{1}(\xi) d \xi ;
$$

and so

$$
\int_{|\xi| \leq 2} u_{0}(R \xi) \varphi_{1}(\xi) d \xi \leq C(R, T) \int_{|\xi| \leq 2} \varphi_{1}(\xi) d \xi
$$

where $C(R, T)=C T^{(1+\alpha)(1-\tilde{p})}+C T^{1+\alpha(1-\tilde{p})} R^{-\beta \tilde{p}}$.

Using the estimate

$$
\begin{aligned}
\inf _{|\xi|>1}\left(u_{0}(R \xi)\right) \int_{|\xi| \leq 2} \varphi_{1}(\xi) d \xi & \leq \int_{1<|\xi| \leq 2} u_{0}(R \xi) \varphi_{1}(\xi) d \xi \\
& \leq \int_{|\xi| \leq 2} u_{0}(R \xi) \varphi_{1}(\xi) d \xi
\end{aligned}
$$

in the left-hand side of (7.7), we conclude, after dividing by $\int_{|\xi| \leq 2} \varphi_{1}(\xi) d \xi$, that

$$
\inf _{|\xi|>1} u_{0}(R \xi) \leq C T^{(1+\alpha)(1-\tilde{p})}+C T^{1+\alpha(1-\tilde{p})} R^{-\beta \tilde{p}}
$$

Passing to the limit in (17.8), as $R \rightarrow \infty$, we obtain

$$
\liminf _{|x| \rightarrow \infty} u_{0}(x) \leq C T^{(1+\alpha)(1-\tilde{p})}=C T^{-\frac{2-\gamma}{p-1}} \text {. }
$$

Appendix. In this appendix, we give a proof of Ju's inequality (see Proposition 3.3 in [16]), in dimension $N \geq 1$ where $\delta \in[0,2]$ and $q \geq 1$, for all nonnegative Schwartz functions $\psi$ (in the general case)

$$
(-\Delta)^{\delta / 2} \psi^{q} \leq q \psi^{q-1}(-\Delta)^{\delta / 2} \psi
$$

The cases $\delta=0$ and $\delta=2$ are obvious, as well as $q=1$. If $\delta \in(0,2)$ and $q>1$, using $[5$, Definition 3.2], we have

$$
(-\Delta)^{\delta / 2} \psi(x)=-c_{N}(\delta) \int_{\mathbb{R}^{N}} \frac{\psi(x+z)-\psi(x)}{|z|^{N+\delta}} d z, \quad \text { for all } x \in \mathbb{R}^{N},
$$

where $c_{N}(\delta)=2^{\delta} \Gamma((N+\delta) / 2) /\left(\pi^{N / 2} \Gamma(1-\delta / 2)\right)$. Then

$$
(\psi(x))^{q-1}(-\Delta)^{\delta / 2} \psi(x)=-c_{N}(\delta) \int_{\mathbb{R}^{N}} \frac{(\psi(x))^{q-1} \psi(x+z)-(\psi(x))^{q}}{|z|^{N+\delta}} d z .
$$


By Young's inequality we have

$$
(\psi(x))^{q-1} \psi(x+z) \leq \frac{q-1}{q}(\psi(x))^{q}+\frac{1}{q}(\psi(x+z))^{q} .
$$

Therefore,

$$
(\psi(x))^{q-1}(-\Delta)^{\delta / 2} \psi(x) \geq \frac{-c_{N}(\delta)}{q} \int_{\mathbb{R}^{N}} \frac{(\psi(x+z))^{q}-(\psi(x))^{q}}{|z|^{N+\delta}} d z=\frac{1}{q}(-\Delta)^{\delta / 2}(\psi(x))^{q} .
$$

\section{REFERENCES}

[1] D. Andreucci, A. F. Tedeev, Universal bounds at the blow-up time for nonlinear parabolic equations, Adv. Differential Equations 10 (2005), no. 1, 89-120. MR.2106122 (2005h:35158)

[2] P. Baras, R. Kersner, Local and global solvability of a class of semilinear parabolic equations. J. Differential Equations 68 (1987), no. 2, 238-252. MR892026 (88k:35089)

[3] P. Baras, M. Pierre, Critère d'existence de solutions positives pour des équations semi-linéaires non monotones, Ann. Inst. H. Poincaré Anal. Non Linéaire 2 (1985), 185-212. MR797270 (87j:45032)

[4] M. Birkner, J. A. Lopez-Mimbela, A. Wakolbinger, Blow-up of semilinear PDE's at the critical dimension. A probabilistic approach (English summary), Proc. Amer. Math. Soc. 130 (2002), no. 8, 2431-2442 (electronic). MR1897470 (2002m:60119)

[5] K. Bogdan, T. Byczkowski, Potential theory for the $\alpha$-stable Schrödinger operator on bounded Lipschitz domains, Studia Mathematica 133 (1999), no. 1, 53-92. MR.1671973 (99m:31010)

[6] T. Cazenave, A. Haraux, Introduction aux problèmes d'évolution semi-linéaires, Ellipses, Paris, (1990). MR:1299976 (95f:35002)

[7] M. Chlebik, M. Fila, From critical exponents to blow-up rates for parabolic problems, Rend. Mat. Appl. (7) 194 (1999), 449-470. MR.1789482 (2001j:35136)

[8] T. Cazenave, F. Dickstein, F. D. Weissler, An equation whose Fujita critical exponent is not given by scaling, Nonlinear Analysis 68 (2008), 862-874. MR2382302 (2009c:35218)

[9] S. Chandrasekhar, Stochastic problems in physics and astronomy, Rev. Mod. Phys. vol. 15 (1943), 1-89. MR0008130 (4:248i)

[10] S. D. Eidelman, A. N. Kochubei, Cauchy problem for fractional diffusion equations, J. Differential Equations 99 (2004), no. 2 211-255. MR.2047909 (2005i:26014)

[11] M. Fila, P. Quittner, The blow-up rate for a semilinear parabolic system, J. Math. Anal. Appl. 238 (1999), 468-476. MR.1715494 (2000g:35095)

[12] A. Fino, G. Karch, Decay of mass for nonlinear equation with fractional Laplacian, J. Monatsh. Math. (2010) 160 375-384.

[13] H. Fujita, On the blowing up of solutions of the problem for $u_{t}=\Delta u+u^{1+\alpha}$, J. Fac. Sci. Univ. Tokyo 13 (1966), 109-124. MR0214914 (35:5761)

[14] M. Guedda, M. Kirane, Criticality for some evolution equations, Differential Equations 37 (2001), 511-520. MR1854046 (2002h:35028)

[15] B. Hu, Remarks on the blow-up estimate for solutions of the heat equation with a nonlinear boundary condition, Differential Integral Equations 9 (1996), 891-901. MR.1392086 (97e:35092)

[16] N. Ju, The maximum principle and the global attractor for the dissipative 2-D quasi-geostrophic equations, Comm. Pure. Anal. Appl. (2005), 161-181. MR2123380 (2005m:37194)

[17] A. A. Kilbas, H. M. Srivastava, J. J. Trujillo, Theory and Applications of Fractional Differential Equations, North-Holland Mathematics Studies, 204. Elsevier Science B.V., Amsterdam, 2006. MR:2218073(2007a:34002)

[18] M. Kirane, Y. Laskri, N.-e. Tatar, Critical exponents of Fujita type for certain evolution equations and systems with spatio-temporal fractional derivatives, J. Math. Anal. Appl. 312 (2005), 488-501. MR2179091 (2006d:35131)

[19] M. Kirane, M. Qafsaoui, Global nonexistence for the Cauchy problem of some nonlinear reactiondiffusion systems, J. Math. Anal. Appl. 268 (2002), 217-243. MR1893203 (2004a:35123)

[20] K. Kobayashi, On some semilinear evolution equations with time-lag, Hiroshima Math. J. 10 (1980), 189-227. MR.558855 (81e:35060)

[21] N. V. Krylov, Lectures on Elliptic and Parabolic Equations in Hölder Spaces, Amer. Math. Soc. (Graduate Studies in Mathematics) v. 12, 1996. MR.1406091(97i:35001) 
[22] O. A. Ladyženskaja, V. A. Solonnikov, N. N. Ural'ceva, Linear and Quasilinear Equations of Parabolic Type, Translations of Mathematical Monographs, vol. 23, Amer. Math. Soc., Providence, RI, 1967. MR0241822 (39:3159b)

[23] E. Mitidieri, S. I. Pohozaev, A priori estimates and blow-up of solutions to nonlinear partial differential equations and inequalities, Proc. Steklov. Inst. Math. 234 (2001), 1-383. MR1879326 (2005d:35004)

[24] M. Nagasawa, T. Sirao, Probabilistic treatment of the blowing up of solutions for a nonlinear integral equation, Trans. Amer. Math. Soc. 139 (1969), 301-310. MR0239379 (39:736)

[25] W. E. Olmstead, private communication.

[26] C. A. Roberts, W. E. Olmstead, Blow-up in a subdiffusive medium of infinite extent, Fract. Calc. Appl. Anal. 12 (2009), no. 2, 179-194. MR2498365 (2010d:35381)

[27] I. M. Sokolov, J. Klafter, From diffusion to anomalous diffusion: a century after Einstein's Brownian Motion, Chaos 15 (2005), 6103-6109. MR2150232 (2006d:82060)

[28] S. G. Samko, A. A. Kilbas, O. I. Marichev, Fractional integrals and derivatives, Theory and Applications, Gordon and Breach Science Publishers, Yverdon, 1987. MR.1347689 (96d:26012)

[29] P. Souplet, Blow-up in nonlocal reaction-diffusion equations, SIAM J. Math. Anal. 29 (1998), 13011334. MR 1638054 (99h:35104)

[30] P. Souplet, Monotonicity of solutions and blow-up for semilinear parabolic equations with nonlinear memory, Z. Angew. Math. Phys. 55 (2004), 28-31. MR2033858 (2004k:35199)

[31] K. Yosida, Functional Analysis, sixth edition, Springer-Verlag, Berlin, 1980. MR617913 (82i:46002) 